\title{
Hodgkin Lymphoma: Recent Progress in Overall Management
}

\author{
Tarec Christoffer El-Galaly ${ }^{\bullet}$ - Anne Segolène Cottereau ${ }^{2} \bullet$ \\ Adalgisa Condoluci i,4,5 • Davide Rossi ${ }^{3,4,5} \bullet$ Jan Maciej Zaucha ${ }^{6}$ \\ Anna Sureda ${ }^{7}$ Bruce Cheson ${ }^{8} \bullet$ Andrea Gallamini ${ }^{9}$ \\ ${ }^{1}$ Hematology Department, Aalborg University Hospital, Denmark; ${ }^{2}$ Nuclear Medicine \\ Department Cochin Hospital, Paris, France; ${ }^{3}$ Laboratory of Experimental Hematology, \\ Institute of Oncology, Bellinzona, Switzerland; ${ }^{4}$ Clinic of Hematology, Oncology Institute \\ of Southern Switzerland, Bellinzona, Switzerland; ${ }^{5}$ Faculty of Biomedical Sciences, Italian \\ Swiss University, Lugano, Switzerland; ${ }^{6}$ Department of Hematology and Bone marrow \\ Transplant, Medical University, Gdansk, Poland; ${ }^{7}$ Clinical Hematology Department Catalan \\ Institute of Oncology - Hospitalet, Barcelona, Spain; ${ }^{8}$ Scientific advisory board Lymphoma \\ Research Foundation, New York, NY, USA; ${ }^{9}$ Research and clinical innovation department \\ A. Lacassagne Cancer Center, Nice, France
}

Author for correspondence: Andrea Gallamini, Research and clinical innovation department, Antoine Lacassagne Cancer Center, Nice, France. Email: andreagallamini@ gmail.com; andrea.GALLAMINI@nice.unicancer.fr

Cite this chapter as: El-Galaly TC, Cottereau AS, Condoluci A, Rossi D, Zaucha JM, Sureda A, Cheson B, Gallamini A. Hodgkin Lymphoma: Recent Progress in Overall Management. In: Gallamini A, Juwied M. editors. Lymphoma. Brisbane (AU): Exon Publications. Online first 2021 Nov 21.

Doi: https://doi.org/10.36255/exon-publications.lymphoma.2021.management

In: Gallamini A, Juwied M (Editors). Lymphoma. Exon Publications, Brisbane, Australia. ISBN: 978-0-6453320-0-1; Doi: https://doi.org/10.36255/exon-publications.lymphoma.2021 Copyright: The Authors.

License: This open access article is licenced under Creative Commons Attribution-NonCommercial 4.0 International (CC BY-NC 4.0) https://creativecommons.org/licenses/by-nc/4.0/ 


\begin{abstract}
The overall approach to the management of Hodgkin lymphoma has undergone a rapid revolution. The Ann Arbor staging, proposed more than half a century ago, is still valid to guide treatment intensity, but all the invasive methods originally proposed have been replaced by a single high-performing tool of functional imaging: the Positron Emission Tomography coupled with ComputedTomography (PET/CT). Apart from improving the overall accuracy of the Ann Arbor staging, new PET-derived metrics to measure the tumor burden such as metabolic tumor volume, total lesion glycolysis and tumor spread such as the Tumor Distance (Dmax), are ready to take over the classical four-level lymphoma staging. PET/CT has also downsized the role of radiation in the classic "combined modality treatment" for HL. PET/CT performed early during treatment (interim PET) for advanced-stage HL remains the standard of care in Europe to escalate treatment in patients starting with ABVD or to de-escalate treatment for patients starting with BEACOPP escalated. Finally, the therapeutic offer for patients failing first-line chemotherapy has been completely renewed by the advent of new nonchemotherapy agents such as Brentuximab Vedotin and Immune Checkpoint Inhibitors (CPI) which are now the standard of care along with autologous stem cell transplant (ASCT).
\end{abstract}

Keywords: CD47 checkpoint; circulating tumor DNA; immune checkpoint inhibitors; management of Hodgkin lymphoma; PET/CT

\title{
INTRODUCTION
}

The management of Hodgkin Lymphoma (HL) has undergone a paradigm shift in recent years. This chapter provides an overview of the recent progress in the clinical management of HL. The authors first focus on the progress achieved by the introduction of the Positron Emission Tomography coupled with ComputedTomography (PET/CT) in the pretherapeutic workup for staging and prognostication. This is followed by a section on the role of cell-free DNA detected in the plasma of patients to assess treatment response and predict the impending disease relapse. The third and the fourth sections summarize recently published results of clinical trials in early and advanced stage HL. The fifth section reports the results of rescue treatment with standard chemotherapy followed by autologous stem cell with or without new drugs, and the sixth section is dedicated to immune checkpoint inhibitors.

\section{PET/CT: A STANDARD TOOL FOR PERSONALIZED HL TREATMENT}

In a disease with cure rate exceeding $85 \%$ as in $\mathrm{HL}$, where a significant proportion of the patients are below the age of 40 years at diagnosis, choosing the therapy with the optimal balance between efficacy and toxicity is critical for long-term outcomes. More specifically, it is key to deliver a therapy that leads to cure in the majority of patients, while at the same time being cognizant of the fact that the 
younger HL survivors with residual life expectancy almost identical to the ageand sex matched background population will endure a long life with the possible consequences of chemo- and radiotherapy, such as secondary cancers and cardiovascular disease (1).

Currently, there is enthusiasm for personalized therapies in hematology and in oncology which are believed to bring therapies with improved benefit/risk balance to patients through tailoring treatments to patient and disease genotype as well as phenotype (2). Within lymphomas, HL is at the forefront of personalized therapy through a long-standing tradition for making treatment decisions based on disease characteristics. The Ann Arbor staging classification, which was originally intended for guidance on staging procedures considered necessary for optimal identification of the patients curable by radiotherapy and those with disseminated disease who had little chance of cure at that time, remains a key component of management of HL patients and is the main determinant in treatment decision (3). However, in the last 2 decades, the prognosis of HL improved tremendously, and a wide range of second line therapy options have led to further improvements (4-6), and staging procedures have been revolutionized by the advances in technology. Today, 18F-flourodeoxyglucose positron/computed tomography (PET/CT) has replaced stand-alone computed tomography (CT) as the preferred imaging modality for staging, as well as early and end-of-therapy response assessments, a practice supported by consensus guidelines for imaging in lymphoma $(7,8)$.

For staging, PET/CT has several advantages over stand-alone CT or PET, as it integrates detailed mapping of glucose metabolism throughout the body with detailed anatomy provided by the CT component. Since active HL lesions unequivocally have increased glucose metabolism, all HL patients with active disease at baseline will display PET-positive lesions (as exception being patients with surgical removal of a single tumor). As in other lymphoma subsets, it has been confirmed that $100 \%$ of HL patients have PET/CT lesions at staging (9). Importantly, PET/CT can identify lesions in areas without clear morphological changes, such as extra nodal involvement or in lymph nodes that are not markedly enlarged. It is estimated that the use of PET/CT over CT results in upstaging in 14-28\% and downstaging in around 5\%, with resulting change in treatment in a substantial proportion of the patients (10-12). The tendency to upstage more patients than downstage has led to stage migration towards higher disease stages in population-based studies (13).

No randomized studies have been performed comparing the outcomes of patients where treatment selections occurred with or without the information provided by PET/CT. In the pre-PET/CT era, bone marrow involvement rate in HL was considered relatively rare compared to rates reported in non-Hodgkin lymphoma. The widespread use of PET/CT has changed this perception and bone marrow involvement represented by focal FDG-avid lesions on PET/CT is observed in around $20 \%$ of the patients, a four-fold increase compared to the $5 \%$ typically reported when assessing only with bone marrow biopsies (Figure 1). Very few patients will have bone marrow involvement in the setting of a positive bone marrow biopsy and negative PET/CT, and clinically relevant upstaging, as a result of bone marrow involvement by biopsy alone, is exceedingly rare $(14,15)$. Therefore, the need for invasive staging in HL has been completely abandoned and PET/CT is the only disease staging procedure necessary prior to therapy. The fact that focal 


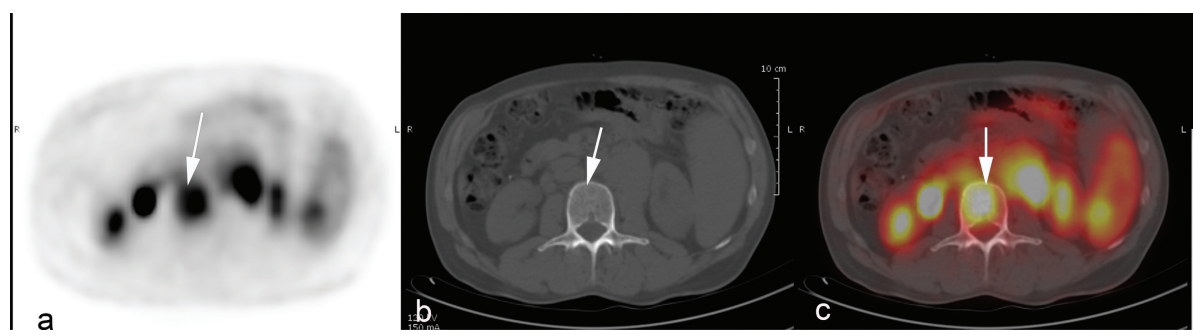

Figure 1. An HL patient with focal lesions in the bone marrow that are not visible of CT. This is one of the most frequent reasons for PET-ascertained upstaging to stage IV disease.

FDG-avid bone marrow lesions are reported to be associated with worse outcomes suggest that the additional findings by PET/CT add relevant prognostic information $(16,17)$. With a first peak in HL incidence around the age of 15-34 years, the large proportion of young patients that are exposed to ionizing radiation is a greater concern than in most other malignancies that occur in older age groups (18). Using a contrast enhanced CT leads to 3 times higher radiation exposure as using a low-dose CT, but using contrast enhanced CT component in PET/CTs rarely changes disease stage or management strategy in lymphoma (19-21). Therefore, a contrast-enhanced CT component can be reserved to patients where the added diagnostic value is needed for other medical reasons (7). Another important aspect of contrast-enhanced CT component, in particular in the treatment response assessment setting, is that contrast fluids lead to stronger SUV increase in the liver and mediastinal blood pool as compared to lymphoma tissue (22). As the visual liver to lesion FDG uptake ratio is now the standard way of assessing metabolic response in tumors, this change may have important clinical consequences.

The greatest advantage of PET/CT in lymphoma is that it can accurately separate residual, viable lymphoma from scar tissue, which is often present after therapy (23). The ability to early identify tumor response before tumor shrinkage makes PET/CT as useful tool for early treatment evaluation. Early metabolic response by PET/CT after few cycles of chemotherapy was shown to be highly prognostic for progression-free survival (PFS), with PET-positive patients after two cycles of ABVD having 2-year PFS of 0\% versus $96 \%$ for those who were PETnegative at the same time point (Figures 2 and 3) (24). This was subsequently confirmed in a larger international series (25). These observations spurred interest in using PET/CT to guide treatment based on early response in both escalation and de-escalation trials $(4,26,27)$. These clinical trials have paved the way for more personalized treatment strategies in HL and are described in detail in the sections about management of early- and late-stage HL.

A common language for assessing metabolic response by PET/CT is necessary to ensure that the treatment decisions are reproducible. The international harmonization criteria from 2007 proposed a dichotomous scoring system where mediastinal blood pool activity was recommended as the reference background activity to define PET positivity for a residual mass greater or equal to $2 \mathrm{~cm}$ in greatest transverse diameter, whereas background update was to be used for assessment of smaller lesions (28). However, dichotomous scores in lymphoma proved suboptimal. 

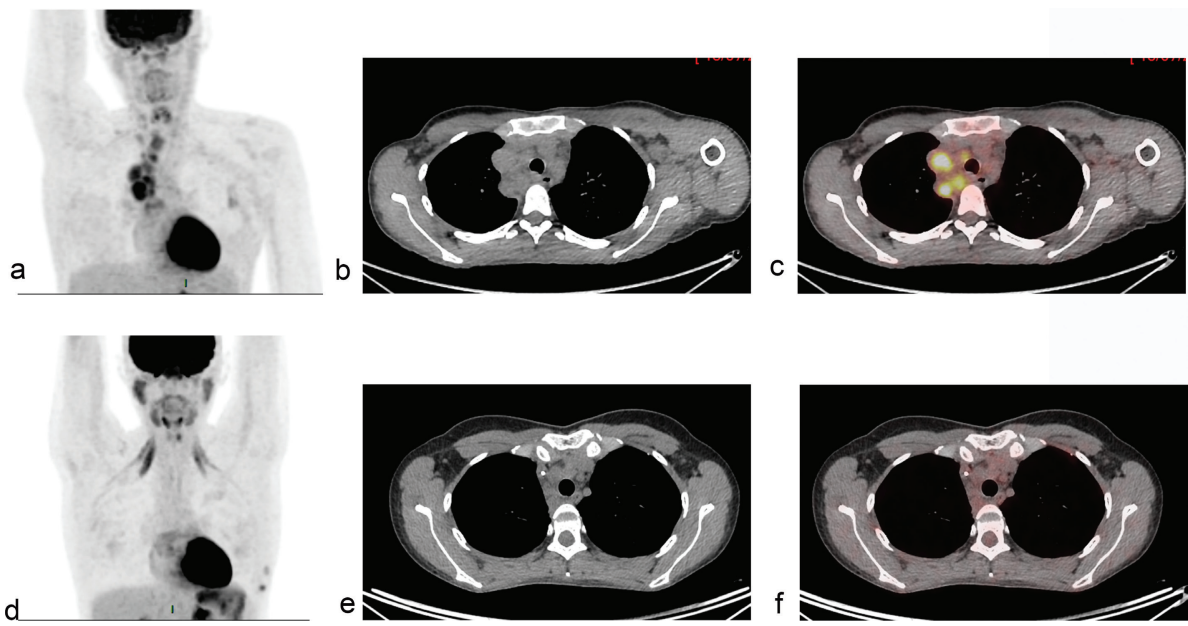

Figure 2. A young woman with a history of progressive swellings, dyspnea, and fever. Biopsy revealed Hodgkin lymphoma. Abnormal FDG-uptake at baseline PET/CT $(\mathbf{a}, \mathbf{b}, \mathbf{c})$ was confined to enlarged clavicular and mediastinal lymph nodes, i.e., disease stage 2. Interim PET/CT $(\mathbf{d}, \mathbf{e}, \mathbf{f})$ after two cycles of ABVD (doxorubicin, bleomycin, vinblastine, and dacarbazine) was coherent with complete metabolic response (Deauville Score 2) although lymph nodes remained enlarged on CT scan.

a
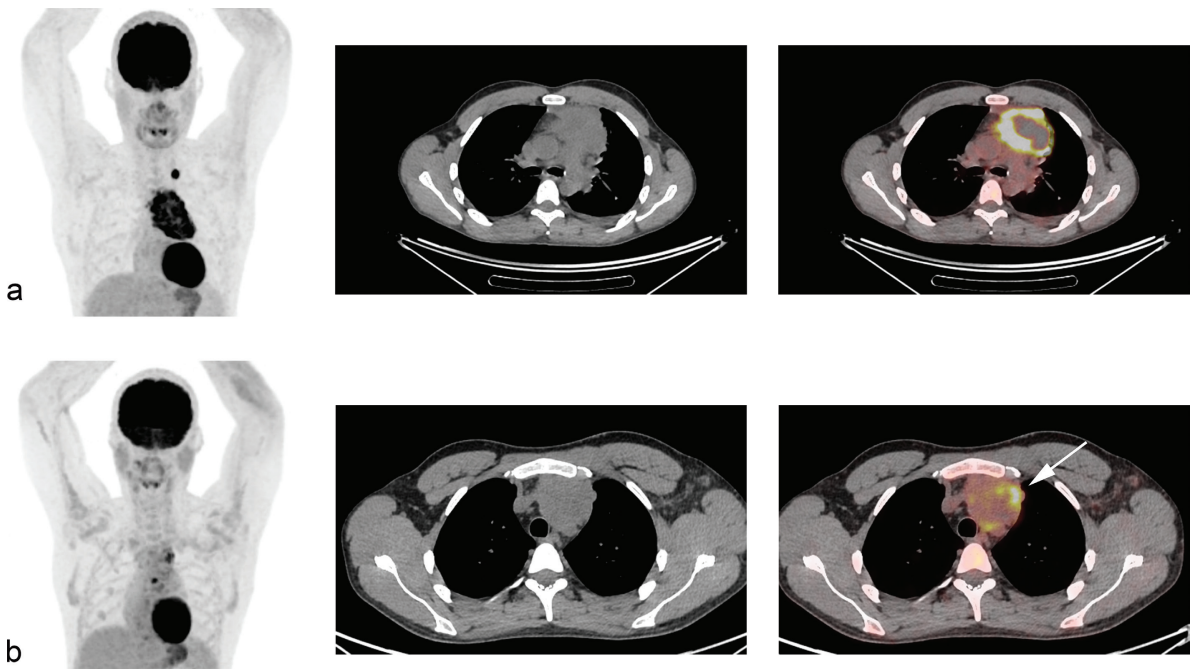

Figure 3. A young woman with Hodgkin lymphoma, stage II. Baseline FDG PET/CT (a). Interim PET/CT (b) performed after two cycles of ABVD (doxorubicin, bleomycin, vinblastine, and dacarbazine,) revealed partial metabolic response with residual FDG-uptake above liver uptake (lymphoma SUVmax=8.6, liver SUVmax=2), classified as DS 4. 
Response-adapted treatment strategies based on interim PET/CT should enable tailoring the risk of false positive/false negative assessments to the question being asked in the trial, which can be done using more granular response reporting (29). The Deauville 5-point score (DS) is now the standard for reporting PET/CT response assessment in lymphoma (Figure 2 and 3) (7). DS was conceived to graduate the intensity of ${ }^{18} \mathrm{~F}$-Fluoro-deoxy-glucose (FDG) uptake in previously identified lymphoma lesions relative to the liver uptake. DS 1 and 2 represent a FDG uptake lower than that of liver, uptake DS 3 represents an intensity of uptake up to that of liver, and DS 4 and 5 represent different degrees of FDG uptake above liver level and new lesions (score 5) (7). For interim and end-of-therapy response assessment in HL, a scores between 1 and 3 are conventionally considered as complete metabolic response (Figure 2) whereas DS 4 or 5 represent active HL (Figure 3) (7). Several studies have shown that the Deauville score is high prognostic in HL using DS 1-3 to define a complete metabolic response (CMR) and DS 4-5 as non-response. In two recently finalized trials of PET-guided deescalation strategies (HD16 and D18) conducted by the German Hodgkin Study Group, the trials were designed to use DS 1-2 (above the mediastinum) as negative (or equivalent), but further analyses of these trials showed that residual uptake above liver level (DS 4-5) was better in predicting outcomes (30-32). In the HD16, 5-year PFS was 93.2\% among PET-2-negative patients and $88.4 \%$ in PET-2-positive patients $(\mathrm{P}=.047)$ when using DS3 + as definition of a positive PET/CT, but when using liver cutoff (DS 4+) for PET-2 positivity, 5-year PFS was 93.1\% for PET negative patients vs $80.9 \%$ for PET positive patients $(\mathrm{P}=.0011)$ (30). In post-hoc analyses of HD18, DS4 was the only risk factor for inferior overall survival in a cohort of patients with DS 1-4 after two cycles of BEACOPP escalated after adjustments for other risk factors (32). The timing of interim PET/ CT in HL is conventionally after two courses of chemotherapy, but a negative PET/ CT after 1 cycle also has a very high negative predictive value (2-year PFS 98\%) and is currently used in clinical trials of very early PET/CT adapted treatment strategies (NCT03517137) (33).

When using interim PET/CT for guiding treatment decisions, high interobserver agreement is critical to ensure consistent treatment decisions. The interagreement of dichotomized reading (DS 1-3 vs DS 4-5) was tested in the HOVON84 trial of diffuse large B-cell lymphoma (34). The inter-observer agreements for interim and end of treatment PET/CT were $88 \%$ and 92\%, respectively, but in both situations, agreement on negative readings were significantly higher than for positive readings suggesting that dual-reading is relevant when conducting response-adapted trials (34). Inter-observer agreement on the granular 1-point DS level, however, seems to be rather poor on only $42 \%$ in a study where 100 interim PET/CTs were assessed by five international experts. When testing agreement on dichotomized PET/CT results (DS 1-3 vs DS 4-5), concordance was $86 \%$ making this distinction most reliable for clinical decision making (35). As in the study based on HOVON84, concordance probability was highest for negative scans (DS 1-3). These results confirm at earlier validation study of the DS in adult advanced stage HL, where Independent agreement among reviewers, defined as at least 4 out of 6 reviewers agreed on DS $1-3$ vs DS $4-5$, was reached in $97 \%$ of patients (36).

After completing treatment, a PET/CT is obtained to confirm remission. Most patients with negative interim PET/CT will also have complete remission at the 
end of therapy and have excellent progression-free survival. However, since most patients (typically $>80 \%$ ) will have a negative interim PET/CT, more than half of the patients who eventually experience treatment failure will not be identified by a positive interim PET/CT. In a study of $76 \mathrm{HL}$ patients, the sensitivity and specificity of interim PET/CT were $47 \%$ and $85 \%$, respectively, whereas for end of treatment PET/CT the corresponding numbers were $80 \%$ and $93 \%$. Eight patients with negative int-PET had treatment failure and six of them were identified as non-responders with end-PET. Therefore, end-of-treatment PET/CT remains important in the setting of a negative interim PET/CT (37). Patients who have a positive end of treatment PET/CT after a negative interim PET/CT have worse outcomes than patients salvaged after a positive interim PET/CT (38). For patients who are in remission after end-of-treatment PET/CT, no further imaging is warranted in routine clinical practice. Surveillance PET/CTs are associated with high false positive rates and follow-up strategies including serial routine imaging do not appear to improve patient outcomes $(39,40)$.

With the introduction of more novel therapies for HL, including immunotherapy with PD1 inhibitors, there was a need for response criteria that provided guidance on the interpretation of transient tumor flare phenomenon or pseudoprogressions that can occur in response to increased immunologic activity in tumors. Therefore, The Lymphoma Response to Immunomodulatory Therapy Criteria (LYRIC) was developed. This set of response criteria include the category indeterminate response to account for situations where individual tumor lesions increase in size or activity in the absence of clinical deterioration (41). At first glance these criteria could look like oxymora but how to define a response as indeterminate? The answer is that these cases need to be monitored closely with additional imaging or biopsy to distinguish pseudo progression from true progressions (Figure 4).
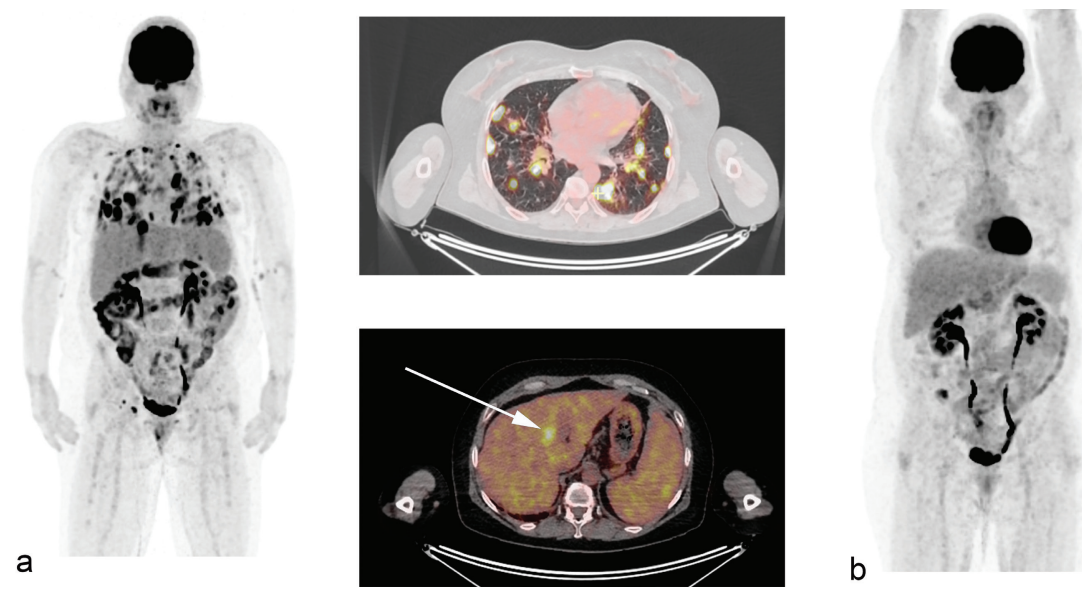

Figure 4. 57-year-old woman with history of $2 \mathrm{HL}$ relapses, the last one treated by Nivolumab. One year after starting nivolumab (PD1 immune check point inhibitor), she developed enlarged mediastinal and abdominal lymph nodes, diffuse pulmonary lesions (SUVmax=27.4) and a hepatic lesion, all with intense FDG uptake A, Biopsies revealed aseptic granuloma including well-formed giant cells consistent with sarcoid-like reaction. PET/CT after corticosteroids (b) showed improvement with a complete metabolic response of all lesions. 


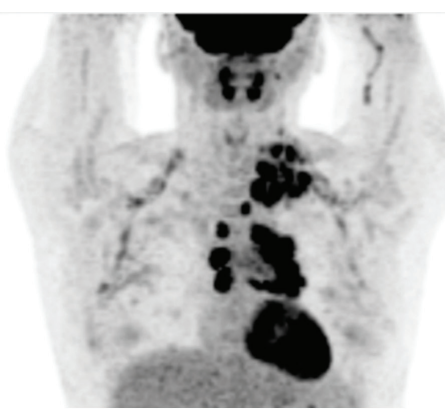

\section{$41 \%$ SUVmax}

a

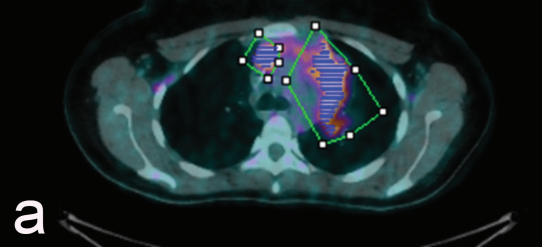

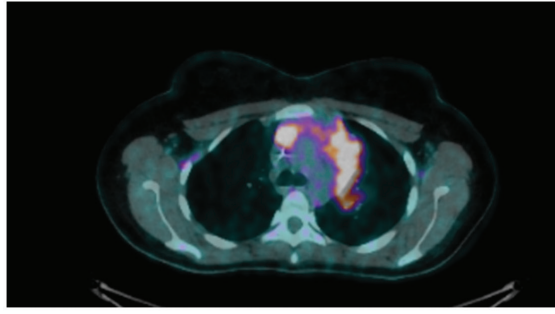

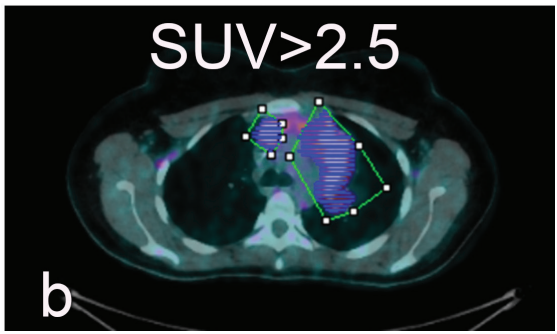

Figure 5. Metabolic tumor volume measurements with volume delineation using the SUV41\% thresholding method (a) and SUV2.5 thresholding method (b). The total metabolic tumor volume (including all the lesions) was $91 \mathrm{ml}$ with the $41 \%$ SUVmax method vs $250 \mathrm{ml}$ with SUV $>2.5$.

Immunotherapies can lead to variable, transient immunologic flares that are shown as increased FDG-uptake and can resemble disease progressions (Figure 4).

In addition to providing important staging information that determines initial therapy, baseline PET/CT can give additional prognostic information. It has been shown that the metabolic tumor volume (MTV) is an important determinant of treatment outcomes in patients with relapsed/refractory HL (Figure 5). Among 65 transplant-eligible patients, predictors of outcomes were baseline MTV and refractory disease in multivariable analyses. For patients with low MTV $\left(<109.5 \mathrm{~cm}^{3}\right)$ and relapsed disease, the 3-year EFS was 100\% (42). MTV is also prognostic in newly diagnosed limited stage HL, as shown in a post-hoc analysis of patients in the EORTC H10 trial. Five-year PFS was $71 \%$ in the high-TMTV $\left(>147 \mathrm{~cm}^{3}\right)$ group vs $92 \%$ in the low-TMTV group $\left(\leq 147 \mathrm{~cm}^{3}\right)$ (43). It is likely that further personalized therapies in HL will be represented by combinations of baseline tumor volumes and early response adaptions.

\section{CIRCULATING TUMOR DNA FOR MONITORING HODGKIN LYMPHOMA}

The genetic landscape of Hodgkin lymphoma (HL) has been historically difficult to explore because of the scarcity of Reed-Sternberg cells in tumor tissue (0.1-3\% 
of tissue cells) (44). Novel next-generation sequencing-based technologies have widely improved the knowledge on HL genetics through the analysis of bloodstream cell tumor DNA (ctDNA), which shows higher median variant allele frequency (VAF, a surrogate measure of the proportion of DNA molecules in the original specimen harboring the mutation) than tissue biopsies and overcomes tumor spatial heterogeneity virtually depicting the whole tumor clonal architecture $(45,46)$.

According to international guidelines (8), the recommended biomarker for disease response assessment in HL is PET/CT, a functional-imaging tool that can anticipate either the cure or the imminent relapse in fluorodeoxyglucose-avid lymphomas $(26,31,47)$. Nevertheless, a portion of these patients will ultimately experience disease progression or relapse. Persistence of ctDNA detection during curative-intent therapy is proposed as a dynamic prognostic marker for clinical outcome that can anticipate and complement interim PET/CT results $(45,48)$.

\section{Sample collection and processing}

The definition of a standardized methodology to collect and process samples is of utmost importance in clinical trials involving ctDNA study, since delay in blood processing, wrong blood storage temperature, or even agitation of the sample can lead to the contamination of ctDNA with germline DNA (gDNA) released from lysed blood cells (49). The development of ctDNA-preserving tubes, containing a formaldehyde-free reagent which stabilizes nucleated blood cells and prevent nuclease-mediated DNA degradation, has considerably improved pre-analytics in this setting (49). DNA extraction, quantification, and quality control must follow validated protocols to warrant uniformity and reliability (45, 48, 50-56). The description of technical aspects of blood processing and ctDNA analysis are beyond the objectives of this chapter and will not be covered here.

\section{ctDNA technologies}

ctDNA is the portion of cell free DNA (cfDNA) which derives from tumor apoptotic cells, and which can be distinguished from cfDNA released by normal cells by using the tumor mutation profile as a fingerprint. Being influenced by disease type and stage, the mean concentration of cfDNA in lymphoma patients is $30 \mathrm{ng} / \mathrm{mL}$, which is up to 3-fold higher compared to that of age/sex matched healthy subjects $(53,57$, $58)$ and show a stereotyped length of nucleosomes ( 147 basis pair: bp) or chromatosomes (nucleosome + linker histone; 167 bp) (59-61).

Lymphoid malignancies are characterized by the expansion of a single clone, with a specific clonal rearrangement of immunoglobulin (Ig) genes (62). The germline variable (V), diversity (D), and joining (J) genes of the IG loci are rearranged during early development phases and undergo random deletion and insertion of nucleotides within the junctional regions, producing a unique sequence for each B lymphocyte (62). Each tumor specific VDJ profile can be considered as a fingerprint to assess clonality, thereby distinguishing malignancy from non-neoplastic processes. When a relapse is suspected, assessment of the clonal relationship between the primary and new lesion can confirm the recurrence. Recent studies on DLBCL patients reported a rate of detection of $82-92 \%$ for Ig 
rearrangement at baseline (pre-treatment timepoint) $(53,58)$. Levels of ctDNA correlated with tumor burden assessed by measuring metabolic tumor volume (MTV) in baseline FDG-PET/CT, and International Prognostic Index (IPI) and could be detected up to 3-3.5 months before overt disease manifestation $(53,58)$. However, the diagnostic and prognostic value of VDJ profile of the heavy chain (IgH) tracking has some disadvantages when applied to HL, including the need for analyzing micro dissected HRS cells. In HL, ctDNA can be explored using two main techniques: polymerase chain reactions (PCR) and next-generation sequencing assays.

\section{Digital PCR (dPCR)}

This is a simple and low-cost tool which needs small amounts of cfDNA to detect a pre-specified mutated region. In a recent retrospective study, the presence of E571K mutation on XPO1, already identified in primary mediastinal B-cell lymphoma, was detected in $24 \%$ of 94 all-stage HL patients (63). Despite its high sensitivity ( $10^{5}$ detection limit), dPCR can be only used for hotspot single-nucleotide variant detection.

\section{Cancer personalized profiling by deep sequencing (CAPP-seq)}

This is an ultrasensitive next-generation sequencing (NGS) technique for cfDNA detection and quantification, allowing deep DNA sequencing at a low ctDNA input level $(52,58)$. CAPP-seq analysis can be modulated according to diseasespecific panels (also known as 'selectors', a set of exonic and intronic targets chosen to cover regions of known recurrent mutations within any particular cancer) to determine the patient's personal tumor mutation profile. With a reported concordance between ctDNA and tissue biopsy of $>80 \%(48,58,64)$, CAPP-seq represents a reliable tool for monitoring Hodgkin and non-Hodgkin lymphomas and it is being incorporated in the design of ongoing clinical trials with the aim of becoming a prognostic tool in clinical practice.

Genotyping HL has been recently gathered using CAPP-seq technology (48). The study reported data from 80 newly diagnosed and 32 refractory patients with HL, including longitudinal samples collected under ABVD chemotherapy and longitudinal samples from relapsing patients treated with chemotherapy and immunotherapy. The proof that ctDNA mirrors HL genetics, derived from the evidence of a high concordance $\left(\mathrm{R}^{2}=0.978\right)$ between CAPP-seq variant, calls for plasma ctDNA and paired tumor of the genomic DNA (gDNA) isolated from HRS cells on biopsy. Biopsy-confirmed tumor mutations were detectable in ctDNA samples with an $87.5 \%$ sensitivity (48). The most commonly mutated genes were STAT6 (37.5\%), TNFAIP3 (35\%), ITPKB (27.5\%), GNA13 (18.7\%), B2M (16.2\%), ATM (15\%), SPEN (12.5\%), and XPO1 (11.2\%). Major pathways emerging as recurrently mutated included NF-kB, PI3K-AKT, cytokine and NOTCH signaling, and immune evasion. For patients under chemotherapy, new mutations may evolve under the clonal evolution process, which is driven by the selective pressure of treatment, and may not be directly causal for relapse. In general, clonal shifts between pretreatment and relapse samples were documented in all cases, demonstrating that evolution after therapy is the rule. Mutations of STAT6, 
GNA13, ITPKB, and TNFAIP3 were preferentially harbored by the ancestral clones, indicating that they are an early event in HL. For patients treated with immunotherapy, the ancestral clones appeared to be eradicated after a few months after treatment initiation, with a concomitant complete reshaping of the clonal landscape and the emergence of new mutations.

Another study of ctDNA of 60 patients with HL at diagnosis reported a median number of variants per patient of 4.2\%, involving SOCS1 (28\%), IGLL5 (26\%), TNFAIP3 (23\%), GNA13 (23\%), STAT6 (21\%) and B2M (19\%). Levels of ctDNA correlated with Lactate Dehydrogenase (LDH), low serum albumin, B-symptoms, International Prognostic Score (IPS) $\geq 3$ and advanced clinical stage (65).

\section{ctDNA and histology}

Four histologic subtypes of HL are described in the WHO classification (66), with similar prognosis and phenotype are indistinctly characterized by the E571K XPO1 recurrent mutation, with no specific restrictions (63). Reed-Sternberg cells express MYC, NOTCH1, and IRF4 in all the histologic HL subtypes, showing no substantial differences (67). Some subtype-specific genetic features have recently been described. STAT6 and TNFAIP3 mutations are more common in nodular sclerosis and EBER-negative HL (48), while B2M mutations have been exclusively reported in nodular sclerosis subtype (68).

\section{ctDNA and outcome}

Risk-tailored treatment strategies need reliable tools to identify high-risk patients and to detect early disease relapse. Pre-treatment ctDNA levels in cHL patients showed a significant correlation with total metabolic tumor volume (TMTV) measured in the baseline PET/CT ( $\mathrm{p}<.001$ ) (48). In the same study, quantification of ctDNA complemented interim PET in determining residual disease. Clearance of ctDNA in blood anticipated outcome in 24 advanced stage HL patients treated with standard ABVD, with a 2-log reduction after two cycles of frontline chemotherapy predicting complete metabolic response (48). In patients with a positive interim PET despite a 2-log drop in ctDNA, the inflammatory response of the surrounding non-neoplastic tissue accounted probably for the false positive results of interim PET. Conversely, all patients achieving less than 2-log reduction of ctDNA after 2 ABVD courses ultimately relapsed (48). This study provided the concept that ctDNA has prognostic implications in $\mathrm{cHL}$ and may implement the overall accuracy of PET/CT.

\section{Future perspectives}

Extracellular vesicular DNA/miRNA and tumor-educated platelets (TEPs) are some of the tumor-derived molecules that circulate in the bloodstream together with ctDNA, and they represent an important complement to liquid-biopsy diagnostic sensitivity $(69,70)$. Extracellular vesicles (EVs), including exosomes and apoptotic bodies, can contain healthy and tumor-derived DNA and miRNAs, but no clear data are available to date about the proportions of this effect on circulating DNA analysis (71). Some uncertainties remain to be addressed before 
translating the ctDNA technologies into the clinic. One question may regard the detection of a 'molecular relapse', whose role has been already established in other hematologic diseases $(72,73)$, but not yet in the lymphoma setting. At the time of writing this article in October 2021, minimal residual disease (MRD) detection by cfDNA LyV4.0 CAPP-seq assay on peripheral blood samples is being tested in the early-stage favorable Hodgkin Lymphoma (RAFTINGNCT04866654) in low-risk patients treated with chemotherapy alone to predict an impending relapse.

\section{EARLY AND INTERMEDIATE-STAGE DISEASE}

Patients with early-stage Hodgkin Lymphoma (eHL) are not a homogeneous group, and treatment toxicities changed overtime based on chemotherapy and radiation therapy progress. Moreover, the observation that the treatment-related morbidity and mortality in this patient subset tends to occur several years after the completion of the treatment (74), complicate the optimal treatment choice for these patients (75).

The variation in prognosis is wide among patients who have stage I or stage II disease, as defined at the Ann Arbor Conference. Therefore, specific prognostic factors for early-stage HL have been identified and broadly classified as diseaseand host-related. They include the presence of a large mediastinal mass, an elevated sedimentation rate, involvement of multiple nodal sites, extranodal involvement, age $\geq 50$ years, or massive splenic disease (Table 1 ). The fact that just one of them is sufficient to allocate patients in a category of "unfavorable" eHL, and the absence of all the above parameters needed to include patients among the "favorable" eHL, confirms the extreme heterogeneity of these patients (76) - with a cure rate well above $90 \%$ and a mortality for non-cancer related events exceeding the rate of disease progression (77).

Due to the paradox of an excellent immediate disease control and the emergence of late treatment-related toxicities, a predictive tool enabling a tailored therapy and risk of disease relapse could be the ideal solution to this need. ${ }^{18} \mathrm{~F}-\mathrm{FDG}$-PET/CT, performed interim during treatment (iPET) as early as after one or two cycles of chemotherapy, emerged as a powerful predictive tool on longterm treatment outcome for both early and advanced stage $\operatorname{HL}(24,25,78,79)$.

The RAPID trial was launched in 2003 with the objective to assess whether in patients with non-bulky clinical stage IA-II A HL and a negative iPET after three ABVD cycles radiotherapy could be safely omitted (80). After three ABVD cycles, 571 patients underwent interim PET scanning. A total of 420 of the 426 patients with a negative iPET were randomized to involved-field radiotherapy (209 patients) or "No Further Therapy" (NFT: 211 patients). The 5-point Deauville scale was used to interpret the result of iPET: score 1-2 were considered consistent with a negative and score 3 to 5 with a positive iPET. The trial was powered to show a non-inferiority of the NFT versus the standard "Combined Modality Therapy" (CMT) arm, with an accepted inferiority margin of $-7 \%$. After a minimum follow-up of 60 months, the 5-year PFS of the standard arm was 94.6\% (95\% C.I.: 91.5-97.7\%) and therefore the non-inferiority margin was $87.6 \%$. The 5-year PFS of the experimental arm was 90.8\% (95\% C.I. 86.9-94.8\%) but the 


\section{TABLE 1 Current prognostic scores to define favorable or unfavorable early-stage Hodgkin lymphoma}

\begin{tabular}{llll} 
Risk Factors & EORTC & GHLSG & CHDSG \\
\cline { 2 - 4 } & Large mediastinal mass & Large mediastinal mass & Large mediastinal mass \\
Age $>50 \mathrm{y}$ & Extranodal disease & Age $>40 \mathrm{y}$. \\
ESR $>50 \mathrm{~mm}$ & ESR $>50 \mathrm{~mm}$ & ESR $>40$ \\
$\geq 4$ involved regions & $\geq 3$ involved regions & $\geq 3$ involved regions
\end{tabular}

* Absence of all factors or presence of just one defines early favorable or unfavorable HL

CHDSG, Canadian Hodgkin Disease Study Group (HDSG); EORTC, European Organization for the Research and Treatment of Cancer; GHLSG, German Hodgkin Lymphoma Study Group.

non-inferiority margin value fell astride the 95\% CI intervals of the 5-year PFS value of NFT arm and therefore the non-inferiority of NFT treatment strategy could not be demonstrated.

The limit for non-inferiority for the treatment of chemotherapy alone of PET-2 negative patients was higher (10\%) in the EORTC/LYSA/FIL H10 trial (81). In this study, upon treatment stratification in favorable and unfavorable eHL according to EORTC criteria (Table 1), enrolled patients were randomized to interim PET (PET-2)-adapted experimental arms or to a conventional CMT treatment with 3 or 4 ABVD courses plus IFRT, in the favorable or unfavorable strata, respectively. In the experimental arm, the escalation to 2 BEACOPP escalated cycles was planned in case of positive PET-2 (with a DS core of 3 to 5), with a superiority study design, and de-escalation to radiation-free chemotherapy with ABVD in patients with negative- iPET2, (with a DS score of 1-2), with a non-inferiority statistical design (81). The non-inferiority arm was stopped after the pre-planned interim analysis due to a much higher incidence of events in the chemotherapy alone arm compared to the standard CMT arm, indicating the futility of non-inferiority approach (82). Nevertheless, the long-term disease control of PET-2 negative patients treated with chemotherapy alone was relatively good with 5-year PFS of $87.1 \%$ and $89.6 \%$ in favorable and unfavorable group, respectively, while the outcome of patients treated with CMT was significantly superior, with a 5-year PFS of $99.0 \%$ and $92.1 \%$, with an HR 15.8 (95\% CI, 3.8 to 66.1) and 1.45 (95\% CI, 0.8 to 2.5), respectively, favoring CMT over CT alone in both strata. In conclusion, chemotherapy alone was able to cure $90.8 \%$ and $88 \%$ of the iPET negative patients in both studies (81).

Two years later, the German Hodgkin Disease Study Group (GHSD) published the results of the HD 16 trial in early stage, favorable HL (30). Briefly, 1115 patients with eHL in stage I-II without risk factors according to GHSG (large mediastinal mass, extranodal lesions, erythrocyte sedimentation rate $>50 \mathrm{~mm}$. or $>30 \mathrm{~mm}$ in presence of B symptoms, or $\geq 3$ nodal regions, Table 1) were enrolled and treated with 2 ABVD courses followed by an interim PET (PET-2). Patients in the standard arm of the study were then addressed to involved-node Radiotherapy (INRT), whatever the results of PET-2. In the experimental arm, patients with a negative PET-2 were randomly assigned to consolidation INRT or no further treatment (NFT). Patients with a positive PET-2 were treated with INRT. In this study, 
the cutoff for a positive PET-2 was set between Deauville score 2 and 3. The trial was powered to exclude an inferiority of $\geq 10 \%$ in 5 -year PFS of chemotherapy alone with ABVD, compared with CMT in PET-2 negative patients in a noninferiority study design, and to detect a 5-year PFS difference of $\geq 5 \%$ between PET-2-positive and -negative patients receiving CMT. Neither of the two objectives was reached: the 5-year PFS of patients with a negative PET-2 in both arms treated with CMT or NFT was $93.4 \%$ [95\% CI 90.4\% to 96.5\%] versus $86.1 \%$ [95\% C.I. $81.4 \%$ to $90.9 \%$ ], with a delta of $7.3 \%$ [-13.0\% to -1.6\%] and an HR of 1.78 [95\% C.I.: 1.02 to 3.12], but the inferiority margin of $83.4 \%$ fell astride the 95\% CI of the 5-year PFS of patient treated with CT alone. When the cutoff for a positive PET-2 was more correctly set between Deauville score 3 and 4, the 5-year PFS of patients treated with CMT and a negative PET-2 was 93.1\% [95\% CI 90.7\% to $95.5 \%$ ] and for patients with a positive PET-2 it was $80.9 \%$ [95\% CI $72.2 \%$ to $88.7 \%]$, with a difference of $-12.2 \%$ [-21.3\% to $-3.1 \%]$, thus allowing a reach of the second study end-point, as the difference of 5-year PFS between the two groups was $12.2 \%$ (> 10\%), with an HR for DS 4 Vs $1-3$ of 2.94 [95\% CI 1.63 to 5.31], $\mathrm{p}=0.0004$.

In these three studies addressed to test the effectiveness of interim PETguided strategy in early favorable disease, the CMT strategy appeared superior, in terms of 5-year PFS, to radiotherapy-free regimens in the immediate disease control, but non-superior in terms of 5-year OS, while the number of events recorded in the CMT arm were even more frequent, albeit in a non-statistically significant way. However, it should be stressed also that while, the intensity of treatment for PET-2 negative patients randomized to NFT in these trials varied of intensity, being limited to 2 ABVD courses in the HD 16 (30), 3 ABVD courses in the RAPID (80) and 4 ABVD courses in the H10 trial (81), the treatment outcome was very similar, being 86\% (5-year PFS), 90.8\% (3-year PFS) and $87.1 \%$ (5-year PFS).

The safety and efficacy of avoiding radiotherapy has been recently explored by the U.S. Alliance group in early-stage HL presenting with a classical bulky and a negative interim PET scan after 2 ABVD cycles (NCT identifier: 01118026). Patients with a negative PET-2 continued with four more ABVD cycles, while patients with a positive PET-2 switched to BEACOPP escalated for 4 cycles followed by Involved-Field Radiotherapy (IFRT). The results of the interim analysis after a minimum follow-up of 36 months after the inclusion of the last patients have been recently presented (83). The 3-yr PFS estimates were 93.1\% (95\% CI: 87.4-99.1\%) in PET-2 negative patients, 89.7\% (95\% CI: 77.2-100.0\%) in PET-2 positive patients (HR=1.01, 85\% upper bound 2.32), and 92.3\% (95\% CI: 87.0 $98.0 \%)$ for all patients. The protocol-defined primary endpoint was met as the PFS hazard ratio for PET2+ vs PET2- was less than 4.1 (one sided $p=0.04$ ). In conclusion, an excellent treatment outcome was observed in all patients using a PET-adapted approach that allowed omission of radiation in $78 \%$ of patients. In addition, PET-2 positive patients treated with escalated BEACOPP, and consolidative RT did not have inferior outcomes.

An alternative approach to the standard treatment of early-stage HL has been recently proposed by Kumar et al. (84) One-hundred-seventeen patients with early-stage unfavorable HL were enrolled, with the following risk factors: $86 \%$ with Memorial Sloan Kettering-defined disease bulk (maximum diameter $>$ $7 \mathrm{~cm}.), 27 \%$ traditional bulk ( $>10 \mathrm{~cm}), 52 \%$ elevated erythrocyte sedimentation 
rate, $21 \%$ extranodal involvement, and $56 \%>2$ involved lymph node sites. The first three cohorts were treated with CMT with Brentuximab-Vedotin and AVD (A-AVD) for four cycles, followed by radiotherapy with different radiation techniques and doses (88 patients) and the fourth cohort (29) with 4 A-AVD courses without radiotherapy. After a median follow-up of 3.8 years $(5.9,4.5,2.5$, and 2.2 years for cohorts $1-4$ ), the overall 2 -year progression-free survival in cohort 1 through 4 was 93\%, 97\%, 90\%, and 97\%, respectively. The authors concluded that the efficacy of BV-AVD supported the safe reduction or even the elimination of consolidative radiation among patients with a negative end-oftreatment PET.

Omitting consolidation radiotherapy in patients with an end-of-treatment negative PET scan was also the object of the HD 17 Trial conducted by the German Hodgkin Disease Study Group (GHDSG). Briefly, 1100 patients enrolled in 224 clinical sites in Germany, Switzerland, Austria, and the Netherlands with at least one of the following risk factors were prospectively enrolled: (i) a classicallydefined bulky mediastinal mass; (ii) extranodal involvement; (ii) either an erythrocyte sedimentation rate of $50 \mathrm{~mm} / \mathrm{h}$ or higher without B-symptoms, or an erythrocyte sedimentation rate of $30 \mathrm{~mm} / \mathrm{h}$ or higher with B-symptoms; or (iv) involvement of three or more nodal areas (85). Patients were then randomly assigned ( $1: 1)$ either to standard combined-modality treatment, in which patients received the 2 BEACOPP escalated cycles plus 2 standard ABVD cycles (so-called " $2+2$ regimen") followed by 30 Gy of involved-field radiotherapy, or to a PETguided treatment group, in which after the $2+2$ regimen, patients underwent involved-node radiotherapy with 30 Gy only in case of a positive end-of therapy PET (PET4) scan. PET4 was available for central blinded review according the 5-point Deauville scale in 979 patients: 486 in the standard combined-modality treatment group (of which 428 included in a per-protocol analysis) and 493 in the PET4-guided treatment group (of which 477 included in a per-protocol analysis).

At a median follow-up of 46.2 months, the 5-year progression-free survival was $97.3 \%$ (95\% C.I. 94.5-98.7) in the standard combined modality treatment group and $95.1 \%$ (95\% C.I. 92.0-97.0) in PET4-guided treatment group (HR 0.523 [95\% C.I. 0.226-1.211]. The between-group difference was $2.2 \%$ (95\% C.I. -0.9 to 5.3 ), a much lower value than the non-inferiority margin of $8 \%$, thus allowing the conclusion that this PET-adapted strategy met the criteria of noninferiority. Moreover, this individualized PET4-guided treatment allowed the omission of radiotherapy in most (nearly 90\%) patients with newly diagnosed early-stage unfavorable Hodgkin lymphoma.

The role of Immune check Point Inhibitors (ICI) in first-line treatment of the early unfavorable HL has been also recently preliminarily assessed in a pilot study by the same group, with impressive results (86). Briefly, 109 early unfavorable patients according to GHSG criteria were enrolled in 35 clinical sites in Germany, and randomized $(1: 1)$ to either concomitant treatment with 4 cycles of nivolumab and AVD (N-AVD) or sequential treatment with 4 doses of nivolumab, 2 cycles of N-AVD, and 2 cycles of AVD at standard doses, followed in both arms by 30-Gy involved-site radiotherapy. Among 101 patients eligible for primary end point analysis, 46 of 51 patients receiving concomitant therapy and 47 of 50 (94\%) patients receiving (90\%) sequential therapy achieved Complete Remission (CR) after study treatment. An unexpectedly high proportion of patients achieved CR 
with nivolumab monotherapy (85\% in the concomitant and 50\% in the sequential arm), and 12-month PFS was 100\% for concomitant, and 98\% (95\% CI, 95\%$100 \%$ ) for sequential treatment, respectively. Overall survival at 12 months was $100 \%$ in both treatment groups.

\section{ADVANCED STAGE HODGKIN LYMPHOMA}

The advanced classical Hodgkin lymphoma (aHL) refers to stage III and IV according to Ann Arbor classification, accounting for about $60 \%$ of newly diagnosed patients (66). However, some intermediate patients with stage II and risk factors such as B symptoms and bulky mediastinum mass or extranodal involvement are also treated in clinical trials or daily practice as the advanced stages $(25,26)$. The unresolved dilemma exists how to avoid overtreatment in those patients as the less intensive and well tolerated ABVD treatment results in a 5-year PFS of approximately $75 \%$ whereas the more intensive escalated BEACOPP provides a PFS rate of $90 \%$ but still without clear benefit for long-term OS $(87,88)$.

Interim PET after 2 chemotherapy cycles (PET-2) proposed in 2007 (25), started a new era of PET response-adapted-treatment strategy in aHL (89). This strategy tried to combine both treatment approaches by offering patients not properly responding to ABVD (PET-2 positive) intensification to escBEACOPP aiming to improve outcomes and reduce the overall toxicity burden. Three prospective intervention trials verifying this hypothesis- the UK RATHL (26), the US Intergroup SWOG0816 (47), and the Italian GITIL/FIL HD0607 (90)—reported a similar rate of PET-2 positivity according to the 5-point Deauville scale (DS score 4 and 5) of 16\%, 18\%, and 19\%, respectively. Small differences might likely result from the differences in patient inclusion since SWOG0816 enrolled only III-IV stage patients whereas UK RATHL and Italian HD0607 included IIA- high risk and IIB or IIB-only patients, respectively. PFS rates at 3-years for PET-2 negative patients were 87\% in Italian HD0607 and 86\% in UK RATHL trial and only 74\% at 5 years $(79 \%$ a t2 years) in SWOG 0816 trial. In addition, RATHL trial showed that in PET-2 negative patients, treatment de-escalation to AVD from $3^{\text {rd }}$ cycle onward did not hamper the efficacy of standard ABVD (91). The HD0607 trial addressed also the vital question related to the consolidative radiotherapy of initially large nodal mass (LNM) defined as a nodal mass with the largest diameter $\geq$ $5 \mathrm{~cm}$ in patients with negative interim and end-of-treatment-PET (DS1-3). The 6-year PFS and OS was not statistically different between patients randomly assigned to receive consolidation radiotherapy to LNM or no further treatment (91\% vs $95 \%$ ). This was confirmed in three cohorts of patients with the largest diameter of LNM measuring 5-7,7-10, and $\geq 10 \mathrm{~cm}$. This finding is practicechanging: avoidance of radiotherapy in patients with aHL and LNM who achieved complete metabolic remission (CMR; roughly about $50 \%$ of aHL patients) should translate into a significant reduction of long-term toxicity (27).

The escalation to escBEACOPP in PET-2 positive patients was partially successful. Indeed, prognosis of these patients improved from 10-35\% who continued ABVD as documented in the observational studies $(24,25,79)$ but only to about $65 \%$ (68\% in the UK RATHL, 66\% in the US SWOG0816 and $60 \%$ in the Italian HD0607 trials). Patients with PET-2 DS5 have still very poor prognosis 
with only 35\% 3-year PFS despite treatment escalation (90). Additionally, escalation to six escBEACOPP cycles in SWOG 0816 instead of four as in two other trials increased the cumulative rate of second cancer to $14 \%$ within the 5.9 years of median follow-up compared to $1.7 \%$ in UK RATHL at 3.4 years whereas in Italian HD0607 no second cancers were reported at 3.6 years median of followup. Clearly, PET response-adapted treatment has disclosed its limitations, in particular for the lower negative predictive value of PET-2 than originally reported and expected with ongoing relapses and other PFS events occurring beyond 2 to 3 years. Despite these limitations, PET response-adapted approach backboned on ABVD is the most important development in the management of aHL in the last decade. PFS of all aHL patients treated in this way improved from about $70 \%$ to $82 \%$ at 3 years in UK RATHL and Italian HD0607 trials and less significantly to $74 \%$ in SWOG0816 and became the standard practice (92).

Alternative approaches have been proposed thanks to the introduction of new effective target agents such as brentuximab vedotin (BV)—antibody-drug conjugate that combines an anti-CD30 antibody with the drug monomethyl auristatin. In ECHELON-1 trial, aHL patients were randomized to receive standard ABVD or AVD plus BV for up to six cycles. This study was not designed with a PET-adapted approach, although a non-decisional PET-2 scan was done and patients with a DS 5 were allowed to be switched to an alternative therapy. The combination of BV + AVD resulted in a superior 5-year modified PFS of $82 \%$ compared with $75 \%$ in the ABVD group regardless of the disease stage at enrolment. Notably, patients with positive PET-2 had 5-year modified PFS of $60 \%$, which is similar to patients escalated to BECAOPP in PET adapted approaches (93). Addition of BV, however, was complicated by peripheral neuropathy, albeit largely reversible and febrile neutropenia requiring G-CSF prophylaxis to lower its incidence from 21 to $11 \%$. Although AVD + BV seem to perform better than standard ABVD (but not better than PET adapted approaches), the costs of upfront treatment of all patients with BV are more than 70 times higher. Alternatively, persistent tumor cell free DNA presence at the time of interim assessment as a biomarker of poor response to ABVD could be proposed to guide treatment intensity (15), however such approach has not been tested as yet.

More promising seems to be the introduction of immune checkpoint inhibitors (CPI) to the first line treatment published by two preliminary reports. The first refers to the Cohort D of the CheckMate 205 trial in which 51 patients with untreated aHL received Nivolumab for four doses, followed by 12 doses of Nivolumab-AVD (94). The objective response rate was $84 \%$ with $67 \%$ achieving complete remission. More importantly with a minimum follow-up of 9.4 months, modified progression-free survival was $92 \%$. The second reports the outcome of 30 patients treated with 3 cycles of pembrolizumab as monotherapy followed by AVD for 4 to 6 cycles, depending on stage and bulk. After pembrolizumab monotherapy, the CMR rate was (37\%) and increased to $100 \%$ after 2 cycles of AVD and was maintained thorough the median follow-up of 22.5 months without any event (95). Such promising results of early administration of CPI before any standard chemotherapy might be explained by the concept of increased sensitization by CPI to chemotherapy, which was first observed in patients with recurrent/resistant disease $(96,97)$. The upcoming time will show whether the early short administration of CPI will alleviate or change PET-response adapted approach in the treatment of aHL. 


\section{RELAPSED OR REFRACTORY CLASSICAL HODGKIN'S LYMPHOMA}

In spite of the high cure rate after first-line conventional chemotherapy (CT) +/radiotherapy, a small proportion of classical Hodgkin's lymphoma (cHL) patients $(5-10 \%)$ are primary refractory and up to $20-30 \%$ of them eventually relapse. High dose CT (HDCT) and autologous stem cell transplantation (auto-HCT) is considered the standard of care for patients failing front-line treatment. Auto-HCT results in a cure rate of about $50 \%$ of the patients $(98,99)$. The most frequently used regimens for second line chemotherapy such as ESHAP (Etoposide, methylprednisolone, High-dose ARA-C, Cisplatin), DHAP (Dexamethasone, High-dose ARA-C, Cisplatin), ICE (Ifosfamide, Carboplatin, Etoposide), and GDP (Gemcitabine, Dexamethasone, Cisplatin) result in an overall response rate (ORR) of $70-80 \%$ and a CR rate of $20-50 \%$. Nevertheless, in these studies, published at the end of the last millennium, the response was evaluated with traditional contrast-enhanced CT scan, making the comparison with the newer combinations in which results were assessed by PET/CT very difficult. No superiority of one over the other regimens has been demonstrated so far. This scenario has been significantly revolutionized by the systematic use of PET/CT. Patients achieving a PET/ CT negative CR (CMR: Complete Metabolic Response) have a significantly higher probability to be alive and disease-free after transplantation than patients showing a persisting FDG-avid lesion auto-HCT (100). The combination of brentuximab vedotin (BV) with different conventional platinum- or gemcitabine-based chemotherapy or with bendamustine (Table 2) (101-104), was able to achieve a CMR in

\begin{tabular}{|c|c|c|c|c|c|c|c|}
\hline \multicolumn{2}{|l|}{ TABLE 2} & \multicolumn{6}{|c|}{$\begin{array}{l}\text { Brentuximab vedotin in combination with } \\
\text { conventional salvage chemotherapies for } \\
\text { patients with classical Hodgkin's lymphoma in } \\
\text { first relapse. }\end{array}$} \\
\hline $\begin{array}{l}\text { Author, } \\
\text { (reference) }\end{array}$ & $\begin{array}{l}\text { N. of } \\
\text { Pts. }\end{array}$ & Protocol & $\begin{array}{l}\text { ORR } \\
(\%)\end{array}$ & $\begin{array}{l}\mathrm{mCR} \\
(\%)\end{array}$ & $\begin{array}{l}\text { N. of Pts. } \\
\text { Undergoing } \\
\text { auto-HCT }\end{array}$ & PFS & OS \\
\hline $\begin{array}{l}\text { Moskowitz } \\
\quad \text { et al. (101) }\end{array}$ & 46 & $\begin{array}{l}\text { Sequential BV+ } \\
\text { Augmented } \\
\text { ICE }\end{array}$ & 80 & 76 & $44(96 \%)$ & $90 \%$ (24 mo) & $95 \%$ (24 mo) \\
\hline $\begin{array}{l}\text { LaCasce et al. } \\
\quad(102)\end{array}$ & 55 & BV + Benda & 92.5 & 74 & $40(75 \%)$ & $70 \%(24 \mathrm{mo})$ & $95 \%$ (24 mo) \\
\hline $\begin{array}{l}\text { García Sanz } \\
\text { et al. (103) }\end{array}$ & 66 & BV + ESHAP & 94 & 70 & $62(94 \%)$ & $70 \%$ (18 mo) & $90 \%$ (18 mo) \\
\hline $\begin{array}{l}\text { Kersten et al. } \\
\quad(104)\end{array}$ & 55 & BV + DHAP & 90 & 81 & $47(90 \%)$ & $74 \%(24 \mathrm{mo})$ & $95 \%$ (24 mo) \\
\hline
\end{tabular}

auto-HCT, autologous stem cell transplantation; Benda, bendamustine; BV, brentuximab vedotin; DHAP, dexamethasone, high-dose Ara-c, cisplatin; ESHAP, etoposide, methylprednisolone, high-dose Ara-c, cisplatin; ICE, ifosfamide, carboplatin, etoposide; mCR, metabolic complete remission; Mo, months; ORR, overall response rate; OS, overall survival; PFS, progression free survival; Pts, Patients. 
up to $70 \%$ of the cases, which seems undoubtedly a significant step forward in achieving a durable second remission compared to previous report in which the response was assessed by conventional radiologic tools. Chemotherapy-free combinations, e.g., BV and the anti-PDl checkpoint inhibitors, were also able to achieve a high-rate complete metabolic response (CMR) resulting in long-term disease control after transplant without the early and late onset toxicity associated to chemotherapy (105).

The evidence of a variable long-term disease control after auto-HCT paved the way to develop strategies to consolidate transplant results for patients with high risk of relapse. In addition to the response to the second line chemotherapy, primary refractory disease, stage IV disease at relapse, ECOG-status $>1$, and a nodal lesion $>5 \mathrm{~cm}$ at relapse were identified as independent risk factors for auto-HCT outcome in a large retrospective multicenter cooperative consortium-the RISPACT consortium (106). In the AETHERA trial (107), patients with at least one of the following risk factors: primary refractory disease, early relapse (duration of CR after autoHCT $<12$ months) and late relapse with extranodal disease were randomized 1:1 to receive either 16 doses of BV single drug or placebo. Consolidation with BV resulted in a significant improvement of disease control in patients with high-risk disease. Based on these results, BV was approved as consolidation therapy for cHL patients with increased risk of relapse after auto-HCT. CPI, either alone (108) or in combination with BV (109) proved also as an effective and well tolerated consolidation strategy after auto-HCT; nevertheless, the low number of patients included in the trials and the short follow-up precludes definitive conclusions. High risk patients may also benefit from tandem autoHCT. However, this strategy, which is feasible and effective as demonstrated by the French group (110) is less frequently used due to the wide variety of targeted therapy-based treatment options in this setting.

The outcome of cHL patients not candidates to auto-HCT because of old age, comorbidities, performance status or refractory disease after first-line salvage CT is quite poor as both the rate and duration of response after second line treatment proved disappointingly low. These patients represent a big unmet medical need and should be an immediate target for prospective clinical trials with new drugs.

Relapses after high dose chemotherapy and auto-HCT have been considered for long a event with an ominous prognosis. The introduction of new treatment strategies has allowed to significantly improve OS upon relapse in these patients. A retrospective analysis of the Lymphoma Working Party (LWP) of the European Society for Blood and Marrow Transplantation (EBMT) indicates that OS at 4 years for patients that relapse after auto-HCT was only of $35 \%$ for patients relapsing between 2005 and 2008 compared to $61 \%$ for patients failing auto-HCT between 2015 and 2017 (111). This improvement is partially related to the availability of BV and the CPI nivolumab and pembrolizumab.

The pivotal phase II clinical trial that tested the efficacy and safety of BV monotherapy for up to 16 doses in a 3-weeks schedule in patients progressing or relapsing after auto-HCT included 102 patients (112). BV resulted in an overall response rate of $75 \%$ with $34 \%$ of the patients achieving a CMR after 4 cycles of the drug. A longer follow-up of the trial showed a 5-year PFS of $52 \%$ for patients in CR, thus suggesting the ability of BV to cure a small patient subset in this worse clinical scenario (113). 
Whether BV or a CPI should be used as the first treatment strategy in patients failing or ineligible to auto-HCT has been tested in the recently published KEYNOTE-204 prospective clinical trial (114). Patients were randomized 1:1 to receive either pembrolizumab for 35 doses or BV for 35 doses. Overall, the PFS was superior for pembrolizumab with a mean duration of response 13.2 vs. 8.3 months. The results of this study seem to suggest that patients failing auto-HCT should be first addressed to a rescue treatment with pembrolizumab while BV should be considered only after failure of CPI drugs.

The introduction of CPI has improved even more the long-term outcome of patients relapsing after auto-HCT. HL may have a genetically driven vulnerability to PD-1 blockade due to the almost universal presence of abnormalities at 9p24.1 that lead to an overexpression of PD-L1 and PD-L2 not only in the malignant Reed Sternberg cells but also in the inflammatory cells that constitute the HL microenvironment (115). Pivotal phase II trials for nivolumab and pembrolizumab (CHECKMATE-205 and KEYNOTE-087) in different cohorts of patients, most of them failing both auto-HCT and prior BV therapy have indicated the capacity of both drugs to achieve response rates of about $70 \%$ with a median PFS of 13-15 months $(108,116)$. Although the CR rates are quite low with these drugs, not exceeding $20 \%$, the results in terms of OS (80\% after 2 years) for patients that achieve a CR but also for those patients in PR are encouraging thus pointing toward (i) an inadequacy of standard criteria to assess treatment response in lymphoma after CPI, and (ii) to the particular effectiveness of these drugs in $\mathrm{HL}$ relapse $(97,117)$.

With the introduction of both BV and CPI in the treatment of relapsed/refractory $\mathrm{CHL}$, indication and timing of allo-HCT for relapsing or refractory $\mathrm{CHL}$ has been significantly modified. Allo-HCT still represents the only curative treatment strategy for patients with cHL failing auto-HCT especially in more recent times (117). Nevertheless, in spite the advent of haploidentical HCT with a potential curative effect comparable to that of allo-HCT from identical siblings and matched unrelated donors (118), the number of allografted patients is steadily declining. Combination of new drugs could be the trump card for a less toxic and very active rescue treatment in this setting (119-121).

\section{IMMUNE CHECKPOINT INHIBITORS}

A number of explanations have been offered for the failure of infiltrating T-cells to eliminate the malignant cells in HL (122). A critical observation is that many of these cells express PD-1, a negative regulator of T-cell activation and function. The ligands PD-L1 and PD-L2, are overexpressed in classical HL due to aberrations of chromosome 9p24.1 (containing the PD-L1 and PD-L2 loci) which plays a fundamental role in the pathogenesis of the disease (123). The PD-1/PDL-1 checkpoint creates an immune inhibitory milieu. PD-1 on cytotoxic T cells binds to PDL-1 and PDL-2 expressed on the RS cells, with the result being T-cell exhaustion (124).

The monoclonal antibodies nivolumab and pembrolizumab are check point inhibitors (CPI), which interrupt PD-1 blockade and exhibit marked clinical benefit in HL. The earliest data with a CPI in relapsed or refractory (R/R) HL were 
from the CheckMate 205, as previously reported in this chapter. $(125,126)$. The drug was well tolerated with the most frequent grade 3-4 toxicities being increased lipase (5\%) or aspartate transaminase (ALT) (3\%), and neutropenia (3\%). Ansell et al. (127) recently presented a long-term update of the Checkmate 205 study in $\mathrm{R} / \mathrm{R}$ HL at a median of 58 months. The ORR response rate was $71 \%$ with $21 \% \mathrm{CRs}$ (Cohort A ORR 65\%, CR 32\%; B ORR 71\%, CR 14\%, C ORR 75\%, CR 21\%). The median duration of response (DOR) was $18.2 \mathrm{~m}$ but was $30.3 \mathrm{~m}$ for the CRs and $13.5 \mathrm{~m}$ for the PRs. Overall PFS was $15.1 \mathrm{~m}$ (A-18.3 m, B-14.8 m, C $15.1 \mathrm{~m}$ ); $37.4 \mathrm{~m}$ for CRs and 15.2 for PRs. 5 yr OS 71.4\%. Two important observations were made: first, successful retreatment was possible, especially for patients in CR for longer than one year. Second, 18\% of patients were free of progression at 5 years, raising the possibility of cure.

Chen et al. (128) updated their results with pembrolizumab from the KEYNOTE-087 trial: Cohort $1(\mathrm{n}=69)$ were treated after ASCT and BV with an ORR of $71.9 \%$ and $27.6 \% \mathrm{CR}$; Cohort $2(\mathrm{n}=69)$ ineligible for ASCT and BV treatment failure with ORR $76.8 \%$ and $26.1 \% \mathrm{CR}$, and Cohort $3(\mathrm{n}=60)$ no BV after ASCT ORR $73.3 \%$ and CR 31.7\%. The respective median duration of response (DOR) was $49.6 \%, 26.4 \%$, and $50.7 \%$, respectively. In general, pembrolizumab and nivolumab are considered similar in efficacy and tolerability with selection being primarily based on schedule (pembrolizumab $200 \mathrm{mg} \mathrm{q} 3$ weeks: nivolumab $3 \mathrm{mg} / \mathrm{kg}$ every 2 weeks) and physician preference.

Since both BV and CPIs are approved for R/R HL, an important question is which is the preferred agent. To address this issue, Kuruvilla et al. conducted a randomized comparison in 304 patients (114). PFS was superior with pembrolizumab with a HR of 0.65 (95\% CI 0.48-0.88, $\mathrm{p}=0.0027$ ). Thus, in patients naive for both agents, a CPI would seem to be the preferred first option (Figure 1).

\section{COMBINATION STRATEGIES}

Given the availability of two relatively well-tolerated and active classes of agents, antibody-drug conjugates, and CPIs, combinations and sequences of BV and CPIs alone or with other agents are actively being developed. Herrera et al. (129) reported a phase 1/2 study of the combination of BV and nivolumab in 62 patients with relapsed or refractory HL. Patients received up to 4 cycles of the combination; during the first cycle, BV was administered on day 1 and nivolumab on day 8 . The drugs were given together on day 1 of subsequent cycles. Patients had a median age of 36 years, $60 \%$ with limited stage disease, and no mention of comorbidities. They were considered to be at high risk as $45 \%$ had primary refractory disease and $31 \%$ had relapsed within 1 year of front-line therapy. The CR was $61 \%$ with an ORR rate of $82 \%$. Time-dependent endpoints could not be reliably analyzed as 54 patients went on to ASCT. Advani et al. (105) updated these data in 91 patients who achieved an ORR of $85 \%$ with CMR of $67 \%$. PFS at 36 months was $77 \%$ for all patients and $91 \%$ for those who had ASCT post BV +/- nivo. Relapsed patients fared better than those who had primary refractory disease with 36 months PFS of $90 \%$ and $61 \%$, respectively. The overall survival was $93 \%$ at 36 months. The future of this combination in $\mathrm{R} / \mathrm{R}$ patients is uncertain given the increased use of BV and CPIs in newer front-line regimens. 
Diefenbach et al. (130) combined the anti-CTLA-4 antibody ipilimumab (Ipi) with $\mathrm{BV}$ and/or nivolumab in a phase $1 / 2$ trial for $\mathrm{R} / \mathrm{R}$ patients with cohorts in which patients received BV + Ipi, BV + nivolumab or the triplet. Using a $3 \times 3$ design, they identified the doses with the triplet of nivolumab $3 \mathrm{mg} / \mathrm{kg}$ and ipilimumab $1 \mathrm{mg} / \mathrm{kg}$ with BV $1.2 \mathrm{mg} / \mathrm{kg}$. Additional patients were enrolled into an expansion phase at these doses. BV and nivolumab were given every 3 weeks whereas Ipi was administered every 12 weeks. Of the 64 patients enrolled, the ORR was $76 \%$ in the Ipi group, $89 \%$ in the nivolumab group, and $82 \%$ in the triplet group. Median PFS was 1.2 years in the Ipi group, but it was not reached in the other two cohorts. BV-nivolumab and the triplet are being compared in NCT01896999.

The use of CPIs as part of initial induction is being extensively studied. Reacting to the impressive results with BV-nivolumab in R/R patients, Cheson et al. (131) conducted a phase 2 trial of the combination in 46 previously untreated patients who were either over the age of 60 years or considered ineligible for standard ABVD because of comorbidities. The combination was not as well tolerated as reported in the R/R setting. Best overall response rate was $91 \%$ with $65 \%$ complete metabolic responses (CMR); however, the cycle 8 ORR was $61 \%$ with $48 \%$ CMRs, which was below the projected outcome. These results may have reflected the shorter duration of CPI therapy of only 8 months.

\section{TOXICITY}

The primary toxicities of concern with PD-1 inhibitors relate to the immunemediated properties of the drugs. These adverse effects are mostly grade 1-2 in severity and include thyroid dysfunction (15\%), rash, hepatitis, and pneumonitis (11\%) $(114,126,128)$. In patients whose endocrine disturbance is asymptomatic, the drug may be continued. Other symptomatic adverse events are generally managed with corticosteroids or other immunosuppressive agents. However, severe toxicities result in drug discontinuation in 5-7\% of patients.

Several groups have evaluated either BV, a CPI, or the combination with chemotherapy as initial treatment for HL. Ramchandren et al. (132) reported on 51 adult patients, almost half of whom had an IPI of 3 or greater. Patients were treated with 4 doses of nivolumab at $240 \mathrm{mg}$ followed by 12 doses of N-AVD, every 2 weeks. The ORR was $84 \%$ with $67 \%$ CR. The 9 -month modified-PFS was $92 \%$. There was an apparent correlation between HRS PDL-1 expression and outcome. The role of nivolumab relative to BV as part of initial treatment is being evaluated in SWOG S1826 in which patients receive either BV or nivolumab in combination with standard AVD chemotherapy (Figure 1).

\section{CD47 CHECKPOINT}

Another checkpoint being targeted is $\mathrm{CD} 47$, a transmembrane protein that is ubiquitously expressed and functions as a "don't eat me" signal, protecting normal cells from phagocytosis by macrophages. CD47 is overexpressed on 
hematologic and solid tumors and correlates with prognosis. CD47-SIRP $\alpha$ functions as an immune checkpoint and provides a novel target for anti-tumor antibodies. Two of these are currently available in the clinic. Magrolimab has been studied in a small number of patients with DLBCL and FL with encouraging results (133). TTI-621 has been studied in several trials. Ansell et al. (134) reported 164 patients with B- and T-NHL, 18 of which were in a phase I dose escalation and 148 in expansion cohorts: 35 with rituximab and 4 with nivolumab. The MTD was determined to be $0.2 \mathrm{mg} / \mathrm{kg}$ with thrombocytopenia as the dose limiting toxicity. Other toxicities included fatigue, infusion reactions and chills. In another phase I study including 25 patients with relapsed/refractory lymphoma (NCT03530683), 5 of them with HL, TTI-621 was administered with a modified $3+3$ schema. Only five patients responded and none of them had HL histology (135).

\section{DURATION OF THERAPY}

There is no uniform consensus on the optimal duration of CPI therapy. Overall, nivolumab and pembrolizumab appear to have comparable activity and a similar toxicity profile. Differences include the schedule of administration: every 2-4 weeks for nivolumab, and every 3 or 6 weeks for pembrolizumab. The duration was until disease progression for nivolumab, and until progression or a maximum of 2 years with pembrolizumab, although patients in CR could go off treatment. Those in PR or with SD should continue on therapy as late CRs are observed. Thus, treatment duration can be tailored to patient convenience or need for more careful follow-up. Of note, is that retreatment has been successful in the majority of patients. Following the long-term follow-up of CheckMate 205, the recommendation was made for discontinuation following a year of treatment for patients in a CR. Although BV is recommended as consolidation for high-risk patients following ASCT based on the AETHERA trial (107), there are, as yet no such data available for CPIs.

\section{Assessment of response}

An important observation with the use of CPIs was that they might be associated with pseudo progression, potentially resulting in premature discontinuation of effective therapy. The mechanism has been speculated to be an acute inflammatory response leading to a false positive result on PET-CT scan. As a result, Cheson et al. (41) developed the LYRIC Criteria as a specific modification of the Lugano Classification. They categorized three types of Indeterminate Response (IR): (i) IR-1 in which there is an increase in overall tumor burden (as assessed by the sum of the product of the diameters [SPD]) of $\geq 50 \%$ of up to 6 measurable lesions in the first 12 weeks of therapy, without clinical evidence of clinical deterioration; (ii) IR-2, appearance of new lesions or growth of one or more existing lesion(s) $\geq 50 \%$ at any time during treatment occurring in the context of lack of overall progression ( $<50 \%$ increase) of overall tumor burden as measured by the SPD of up to 6 lesions at any time during the treatment; and (iii) IR3, increase in FDG uptake of one or more lesion(s) without a concomitant increase 
in lesion size or number. A repeat scan in about 12 weeks may allow for distinction between PD and pseudo progression in the absence of a biopsy. It is possible that this distinction may also be facilitated with the measurement of circulating tumor DNA.

\section{THE CURRENT ROLE FOR CPIS IN THE MANAGEMENT OF CLASSICAL HL}

The role for CPIs in the management of patients with classical HL is in evolution (Figure 6). Their role relative to BV as part of initial treatment is being evaluated in SWOG S1826 in which patients receive either BV or nivolumab in combination with standard AVD chemotherapy.

Currently, autologous stem cell transplantation remains a standard for patients with relapsed HL. Nonetheless, the optimal reinduction strategy is controversial. CPIs are being incorporated into salvage regimens to determine if they can increase the number of transplant eligible patients. For patients who are transplant ineligible or progressing post-transplant, $\mathrm{CPI}$ have been shown to be superior to BV (114). For patients who achieve a CR with a CPI, treatment may be discontinued after a year, with retreatment possible following progression. Since some patients may not relapse, at least for a number of years, allogeneic BMT may be deferred until evidence of PD (127). Those who fail to achieve a CR with a CPI can be treated with BV, if BV naive, or referred for allogeneic BMT. It is likely that CAR-T or bispecific antibodies will replace BMT in this setting and will alter this paradigm.

\section{Current Use of CPIs in $\mathrm{CHL}$}

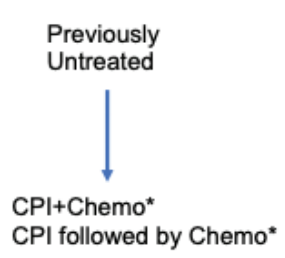

$\mathrm{CPI}+\mathrm{Chemo}^{\star}$
$\mathrm{CPI}$ followed by Chemo*

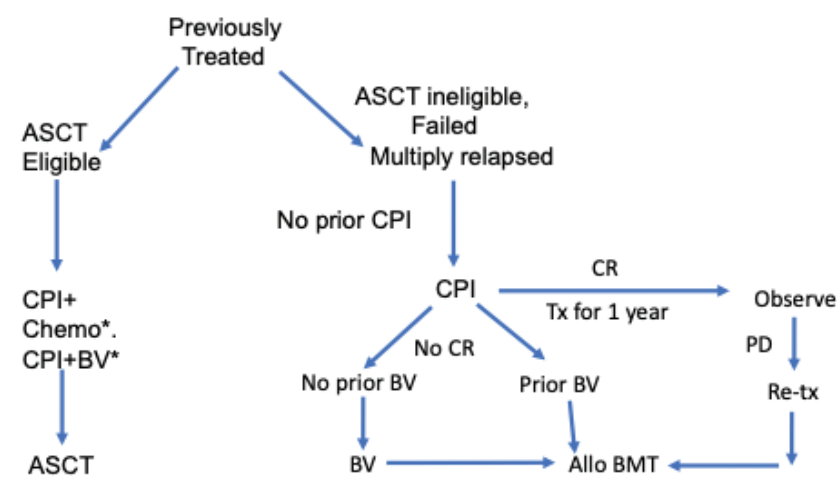

* On a clinical trial

Figure 6. Flow chart depicting the current use of checkpoint inhibitors (CPI) for classical Hodgkin lymphoma. 


\section{CONCLUSION}

In this chapter the most significant advances achieved in the last ten years that have revolutionized the overall management of Hodgkin Lymphoma have been reviewed and critically discussed. Innovative aspects of diagnosis, staging, prognostication, first-line, and rescue treatment and response-assessment monitoring are presented, and their relative advantage are assessed, and compared to the standard approach in use before the introduction of any of them.

Conflict of interest: TEG declares previous employment by Roche (Basel) and speakers fee from AbbVie. The other authors declare no potential conflicts of interest with respect to research, authorship and/or publication of this manuscript.

Copyright and permission statement: The authors confirm that the materials included in this chapter do not violate copyright laws. Where relevant, appropriate permissions have been obtained from the original copyright holder(s), and all original sources have been appropriately acknowledged or referenced.

\section{REFERENCES}

1. Biccler JL, Glimelius I, Eloranta S, Smeland KB, Brown PN, Jakobsen LH, et al. Relapse Risk and Loss of Lifetime After Modern Combined Modality Treatment of Young Patients With Hodgkin Lymphoma: A Nordic Lymphoma Epidemiology Group Study. J Clin Oncol. 2019;37(9):703-13. https://doi. org/10.1200/JCO.18.01652

2. Collins FS, Varmus H. A new initiative on precision medicine. N Engl J Med. 2015;372(9):793-5. https://doi.org/10.1056/NEJMp1500523

3. Carbone PP, Kaplan HS, Musshoff K, Smithers DW, Tubiana M. Report of the Committee on Hodgkin's Disease Staging Classification. Cancer Res. 1971;31(11):1860-1.

4. André MPE, Girinsky T, Federico M, Reman O, Fortpied C, Gotti M, et al. Early Positron Emission Tomography Response-Adapted Treatment in Stage I and II Hodgkin Lymphoma: Final Results of the Randomized EORTC/LYSA/FIL H10 Trial. Journal of Clinical Oncology. 2017;35(16):1786-94. https://doi.org/10.1200/JCO.2016.68.6394

5. Borchmann P, Goergen H, Kobe C, Lohri A, Greil R, Eichenauer DA, et al. PET-guided treatment in patients with advanced-stage Hodgkin's lymphoma (HD18): final results of an open-label, international, randomised phase 3 trial by the German Hodgkin Study Group. Lancet. 2017;390(10114):2790-802. https://doi.org/10.1016/S0140-6736(17)32134-7

6. Guru Murthy GS, Szabo A, Hamadani M, Fenske TS, Shah NN. Contemporary Outcomes for Advanced-Stage Classical Hodgkin Lymphoma in the U.S.: Analysis of Surveillance, Epidemiology, and End Results Database. Oncologist. 2019;24(11):1488-95. https://doi.org/10.1634/ theoncologist.2019-0172

7. Barrington SF, Mikhaeel NG, Kostakoglu L, Meignan M, Hutchings M, Mueller SP, et al. Role of imaging in the staging and response assessment of lymphoma: consensus of the International Conference on Malignant Lymphomas Imaging Working Group. J Clin Oncol. 2014;32(27):3048-58. https://doi. org/10.1200/JCO.2013.53.5229

8. Cheson BD, Fisher RI, Barrington SF, Cavalli F, Schwartz LH, Zucca E, et al. Recommendations for initial evaluation, staging, and response assessment of Hodgkin and non-Hodgkin lymphoma: the Lugano classification. J Clin Oncol. 2014;32(27):3059-68. https://doi.org/10.1200/ JCO.2013.54.8800 
9. Weiler-Sagie M, Bushelev O, Epelbaum R, Dann EJ, Haim N, Avivi I, et al. (18)F-FDG avidity in lymphoma readdressed: a study of 766 patients. J Nucl Med. 2010;51(1):25-30. doi: 10.2967/ jnumed.109.067892. Epub 2009 Dec 15. https://doi.org/10.2967/jnumed.109.067892

10. Hutchings M, Loft A, Hansen M, Pedersen LM, Berthelsen AK, Keiding S, et al. Position emission tomography with or without computed tomography in the primary staging of Hodgkin's lymphoma. Haematologica. 2006;91(4):482-9.

11. Bednaruk-Mlynski E, Pienkowska J, Skorzak A, Malkowski B, Kulikowski W, Subocz E, et al. Comparison of positron emission tomography/computed tomography with classical contrastenhanced computed tomography in the initial staging of Hodgkin lymphoma. Leuk Lymphoma. 2015;56(2):377-82. https://doi.org/10.3109/10428194.2014.919635

12. Barrington SF, Kirkwood AA, Franceschetto A, Fulham MJ, Roberts TH, Almquist H, et al. PET-CT for staging and early response: results from the Response-Adapted Therapy in Advanced Hodgkin Lymphoma study. Blood. 2016;127(12):1531-8. https://doi.org/10.1182/blood-2015-11-679407

13. El-Galaly TC, Hutchings M, Mylam KJ, Brown Pde N, Bukh A, Johnsen HE, et al. Impact of 18F-fluorodeoxyglucose positron emission tomography/computed tomography staging in newly diagnosed classical Hodgkin lymphoma: fewer cases with stage I disease and more with skeletal involvement. Leuk Lymphoma. 2014;55(10):2349-55. https://doi.org/10.3109/10428194.2013.875 169

14. El-Galaly TC, d'Amore F, Mylam KJ, de Nully Brown P, Bogsted M, Bukh A, et al. Routine bone marrow biopsy has little or no therapeutic consequence for positron emission tomography/computed tomography-staged treatment-naive patients with Hodgkin lymphoma. J Clin Oncol. 2012;30(36):4508-14. doi: 10.1200/JCO.2012.42.4036. Epub 2012 Nov 13. https://doi.org/10.1200/JCO.2012.42.4036

15. Voltin CA, Goergen H, Baues C, Fuchs M, Mettler J, Kreissl S, et al. Value of bone marrow biopsy in Hodgkin lymphoma patients staged by FDG PET: results from the German Hodgkin Study Group trials HD16, HD17, and HD18. Ann Oncol. 2018;29(9):1926-31. https://doi.org/10.1093/annond mdy 250

16. Zwarthoed C, El-Galaly TC, Canepari M, Ouvrier MJ, Viotti J, Ettaiche M, et al. Prognostic Value of Bone Marrow Tracer Uptake Pattern in Baseline PET Scans in Hodgkin Lymphoma: Results from an International Collaborative Study. J Nucl Med. 2017;58(8):1249-54. https://doi.org/10.2967/ jnumed.116.184218

17. Pedersen MA, Gormsen LC, Kamper P, Wassberg C, Andersen MD, d'Amore AL, et al. Focal skeletal FDG uptake indicates poor prognosis in cHL regardless of extent and first-line chemotherapy. Br J Haematol. 2019;186(3):431-9. https://doi.org/10.1111/bjh.15933

18. Morton LM, Wang SS, Devesa SS, Hartge P, Weisenburger DD, Linet MS. Lymphoma incidence patterns by WHO subtype in the United States, 1992-2001. Blood. 2006;107(1):265-76. https://doi. org/10.1182/blood-2005-06-2508

19. Quinn B, Dauer Z, Pandit-Taskar N, Schoder H, Dauer LT. Radiation dosimetry of 18F-FDG PET/CT: incorporating exam-specific parameters in dose estimates. BMC Med Imaging. 2016;16(1):41. https:// doi.org/10.1186/s12880-016-0143-y

20. Elstrom RL, Leonard JP, Coleman M, Brown RK. Combined PET and low-dose, noncontrast CT scanning obviates the need for additional diagnostic contrast-enhanced CT scans in patients undergoing staging or restaging for lymphoma. Ann Oncol. 2008;19(10):1770-3. https://doi.org/10.1093/ annonc/mdn282

21. van Hamersvelt HP, Kwee TC, Fijnheer R, Beek FJ, de Klerk JM, Nievelstein RA. Can full-dose contrast-enhanced CT be omitted from an FDG-PET/CT staging examination in newly diagnosed FDG-avid lymphoma? J Comput Assist Tomogr. 2014;38(4):620-5. https://doi.org/10.1097/ RCT.0000000000000067

22. Voltin CA, Mettler J, Boellaard R, Kuhnert G, Dietlein M, Borchmann P, et al. Quantitative assessment of 18F-FDG PET in patients with Hodgkin lymphoma: is it significantly affected by contrast-enhanced computed tomography attenuation correction? Nucl Med Commun. 2019;40(3):249-57. https://doi. org/10.1097/MNM.0000000000000956

23. Engert A, Haverkamp H, Kobe C, Markova J, Renner C, Ho A, et al. Reduced-intensity chemotherapy and PET-guided radiotherapy in patients with advanced stage Hodgkin's lymphoma (HD15 trial): a 
randomised, open-label, phase 3 non-inferiority trial. Lancet. 2012;379(9828):1791-9. https://doi. org/10.1016/S0140-6736(11)61940-5

24. Hutchings M, Loft A, Hansen M, Pedersen LM, Buhl T, Jurlander J, et al. FDG-PET after two cycles of chemotherapy predicts treatment failure and progression-free survival in Hodgkin lymphoma. Blood. 2006;107(1):52-9. https://doi.org/10.1182/blood-2005-06-2252

25. Gallamini A, Hutchings M, Rigacci L, Specht L, Merli F, Hansen M, et al. Early Interim 2-[18F]Fluoro2-Deoxy-D-Glucose Positron Emission Tomography Is Prognostically Superior to International Prognostic Score in Advanced-Stage Hodgkin's Lymphoma: A Report From a Joint Italian-Danish Study. J Clin Oncol. 2007;25(24):3746-52. https://doi.org/10.1200/JCO.2007.11.6525

26. Johnson P, Federico M, Kirkwood A, Fosså A, Berkahn L, Carella A, et al. Adapted Treatment Guided by Interim PET-CT Scan in Advanced Hodgkin's Lymphoma. New England Journal of Medicine. 2016;374(25):2419-29. https://doi.org/10.1056/NEJMoal510093

27. Gallamini A, Rossi A, Patti C, Picardi M, Romano A, Cantonetti M, et al. Consolidation Radiotherapy Could Be Safely Omitted in Advanced Hodgkin Lymphoma With Large Nodal Mass in Complete Metabolic Response After ABVD: Final Analysis of the Randomized GITIL/FIL HD0607 Trial. J Clin Oncol. 2020;38(33):3905-13. https://doi.org/10.1200/JCO.20.00935

28. Juweid ME, Stroobants S, Hoekstra OS, Mottaghy FM, Dietlein M, Guermazi A, et al. Use of positron emission tomography for response assessment of lymphoma: consensus of the Imaging Subcommittee of International Harmonization Project in Lymphoma. J Clin Oncol. 2007;25(5):571-8. https://doi. org/10.1200/JCO.2006.08.2305

29. Meignan M, Gallamini A, Haioun C. Report on the First International Workshop on Interim-PET-Scan in Lymphoma. Leuk Lymphoma. 2009;50(8):1257-60. https://doi.org/10.1080/10428190903040048

30. Fuchs M, Goergen H, Kobe C, Kuhnert G, Lohri A, Greil R, et al. Positron Emission TomographyGuided Treatment in Early-Stage Favorable Hodgkin Lymphoma: Final Results of the International, Randomized Phase III HD16 Trial by the German Hodgkin Study Group. J Clin Oncol. 2019;37(31):2835-45. https://doi.org/10.1200/JCO.19.00964

31. Borchmann P, Haverkamp H, Lohri A, Mey U, Kreissl S, Greil R, et al. Progression-free survival of early interim PET-positive patients with advanced stage Hodgkin's lymphoma treated with BEACOPP $\mathrm{escalted}_{3}$ alone or in combination with rituximab (HD18): an open-label, international, randomised phase 3 study by the German Hodgkin Study Group. The Lancet Oncology. 2017;18(4):454-63. https://doi. org/10.1016/S1470-2045(17)30103-1

32. Kobe C, Goergen H, Baues C, Kuhnert G, Voltin CA, Zijlstra J, et al. Outcome-based interpretation of early interim PET in advanced-stage Hodgkin lymphoma. Blood. 2018;132(21):2273-9. https://doi. org/10.1182/blood-2018-05-852129

33. Hutchings M, Kostakoglu L, Zaucha JM, Malkowski B, Biggi A, Danielewicz I, et al. In Vivo Treatment Sensitivity Testing With Positron Emission Tomography/Computed Tomography After One Cycle of Chemotherapy for Hodgkin Lymphoma. Journal of Clinical Oncology. 2014;32(25):2705-11. https:// doi.org/10.1200/JCO.2013.53.2838

34. Burggraaff CN, Cornelisse AC, Hoekstra OS, Lugtenburg PJ, De Keizer B, Arens AIJ, et al. Interobserver Agreement of Interim and End-of-Treatment (18)F-FDG PET/CT in Diffuse Large B-Cell Lymphoma: Impact on Clinical Practice and Trials. J Nucl Med. 2018;59(12):1831-6. https://doi.org/10.2967/ jnumed.118.210807

35. Kluge R, Chavdarova L, Hoffmann M, Kobe C, Malkowski B, Montravers F, et al. Inter-Reader Reliability of Early FDG-PET/CT Response Assessment Using the Deauville Scale after 2 Cycles of Intensive Chemotherapy (OEPA) in Hodgkin's Lymphoma. PLoS One. 2016;11(3):e0149072. https:// doi.org/10.1371/journal.pone.0149072

36. Biggi A, Gallamini A, Chauvie S, Hutchings M, Kostakoglu L, Gregianin M, et al. International Validation Study for Interim PET in ABVD-Treated, Advanced-Stage Hodgkin Lymphoma: Interpretation Criteria and Concordance Rate Among Reviewers. Journal of Nuclear Medicine. 2013;54(5):683-90. https:// doi.org/10.2967/jnumed.112.110890

37. Mesguich C, Cazeau AL, Bouabdallah K, Soubeyran P, Guyot M, Milpied N, et al. Hodgkin lymphoma: a negative interim-PET cannot circumvent the need for end-of-treatment-PET evaluation. Br J Haematol. 2016;175(4):652-60. https://doi.org/10.1111/bjh.14292 
38. Rigacci L, Puccini B, Broccoli A, Dona M, Gotti M, Evangelista A, et al. Clinical characteristics of interim-PET negative patients with a positive end PET from the prospective HD08-01 FIL study. Ann Hematol. 2020;99(2):283-91. https://doi.org/10.1007/s00277-019-03889-3

39. Jakobsen LH, Hutchings M, de Nully Brown P, Linderoth J, Mylam KJ, Molin D, et al. No survival benefit associated with routine surveillance imaging for Hodgkin lymphoma in first remission: a Danish-Swedish population-based observational study. Br J Haematol. 2016;173(2):236-44. https:// doi.org/10.1111/bjh.13943

40. El-Galaly TC, Mylam KJ, Brown P, Specht L, Christiansen I, Munksgaard L, et al. Positron emission tomography/computed tomography surveillance in patients with Hodgkin lymphoma in first remission has a low positive predictive value and high costs. Haematologica. 2012;97(6):931-6. https:// doi.org/10.3324/haematol.2011.056010

41. Cheson BD, Ansell S, Schwartz L, Gordon LI, Advani R, Jacene HA, et al. Refinement of the Lugano Classification lymphoma response criteria in the era of immunomodulatory therapy. Blood. 2016;128:2489-96. https://doi.org/10.1182/blood-2016-05-718528

42. Moskowitz AJ, Schöder H, Gavane S, Thoren KL, Fleisher M, Yahalom J, et al. Prognostic significance of baseline metabolic tumor volume in relapsed and refractory Hodgkin lymphoma. Blood. 2017;130(20):2196-203. https://doi.org/10.1182/blood-2017-06-788877

43. Cottereau AS, Versari A, Loft A, Casasnovas O, Bellei M, Ricci R, et al. Prognostic value of baseline metabolic tumor volume in early-stage Hodgkin lymphoma in the standard arm of the H10 trial. Blood. 2018;131(13):1456-63. https://doi.org/10.1182/blood-2017-07-795476

44. Schmitz R, Stanelle J, Hansmann ML, Küppers R. Pathogenesis of classical and lymphocyte-predominant Hodgkin lymphoma. Annu Rev Pathol. 2009;4:151-74. https://doi.org/10.1146/annurev. pathol.4.110807.092209

45. Camus V, Viennot M, Lequesne J, Viailly PJ, Bohers E, Bessi L, et al. Targeted genotyping of circulating tumor DNA for classical Hodgkin lymphoma monitoring: a prospective study. Haematologica. 2021;106(1):154-62. https://doi.org/10.3324/haematol.2019.237719

46. Gerlinger M, Rowan AJ, Horswell S, Math M, Larkin J, Endesfelder D, et al. Intratumor heterogeneity and branched evolution revealed by multiregion sequencing. N Engl J Med. 2012;366(10):883-92. https://doi.org/10.1056/NEJMoal113205

47. Press OW, Li H, Schöder H, Straus DJ, Moskowitz CH, LeBlanc M, et al. US Intergroup Trial of ResponseAdapted Therapy for Stage III to IV Hodgkin Lymphoma Using Early Interim FluorodeoxyglucosePositron Emission Tomography Imaging: Southwest Oncology Group S0816. Journal of Clinical Oncology. 2016;34(17):2020-7. https://doi.org/10.1200/JCO.2015.63.1119

48. Spina V, Bruscaggin A, Cuccaro A, Martini M, Di Trani M, Forestieri G, et al. Circulating tumor DNA reveals genetics, clonal evolution, and residual disease in classical Hodgkin lymphoma. Blood. 2018;131(22):2413-25. https://doi.org/10.1182/blood-2017-11-812073

49. Rossi D, Condoluci A, Spina V, Gaidano G. Methods for Measuring ctDNA in Lymphomas. Methods Mol Biol. 2019;1881:253-65. https://doi.org/10.1007/978-1-4939-8876-1_19

50. Cirillo M, Craig AFM, Borchmann S, Kurtz DM. Liquid biopsy in lymphoma: Molecular methods and clinical applications. Cancer Treat Rev. 2020;91:102106. https://doi.org/10.1016/j.ctrv.2020.102106

51. Rossi D, Kurtz DM, Roschewski M, Cavalli F, Zucca E, Wilson WH. The development of liquid biopsy for research and clinical practice in lymphomas: Report of the 15-ICML workshop on ctDNA. Hematol Oncol. 2020;38(1):34-7. https://doi.org/10.1002/hon.2704

52. Newman AM, Bratman SV, To J, Wynne JF, Eclov NC, Modlin LA, et al. An ultrasensitive method for quantitating circulating tumor DNA with broad patient coverage. Nat Med. 2014;20(5):548-54. https://doi.org/10.1038/nm.3519

53. Roschewski M, Dunleavy K, Pittaluga S, Moorhead M, Pepin F, Kong K, et al. Circulating tumour DNA and CT monitoring in patients with untreated diffuse large B-cell lymphoma: a correlative biomarker study. Lancet Oncol. 2015;16(5):541-9. https://doi.org/10.1016/S1470-2045(15)70106-3

54. Bohers E, Viailly PJ, Dubois S, Bertrand P, Maingonnat C, Mareschal S, et al. Somatic mutations of cell-free circulating DNA detected by next-generation sequencing reflect the genetic changes in both germinal center B-cell-like and activated B-cell-like diffuse large B-cell lymphomas at the time of diagnosis. Haematologica. 2015;100(7):e280-4. https://doi.org/10.3324/ haematol.2015.123612 
55. Newman AM, Lovejoy AF, Klass DM, Kurtz DM, Chabon JJ, Scherer F, et al. Integrated digital error suppression for improved detection of circulating tumor DNA. Nat Biotechnol. 2016;34(5):547-55. https://doi.org/10.1038/nbt.3520

56. Abbosh C, Swanton C, Birkbak NJ. Clonal haematopoiesis: a source of biological noise in cell-free DNA analyses. Ann Oncol. 2019;30(3):358-9. https://doi.org/10.1093/annonc/mdy552

57. Hohaus S, Giachelia M, Massini G, Mansueto G, Vannata B, Bozzoli V, et al. Cell-free circulating DNA in Hodgkin's and non-Hodgkin's lymphomas. Ann Oncol. 2009;20(8):1408-13. https://doi. org/10.1093/annonc/mdp006

58. Kurtz DM, Green MR, Bratman SV, Scherer F, Liu CL, Kunder CA, et al. Noninvasive monitoring of diffuse large B-cell lymphoma by immunoglobulin high-throughput sequencing. Blood. 2015;125(24):3679-87. https://doi.org/10.1182/blood-2015-03-635169

59. Giacona MB, Ruben GC, Iczkowski KA, Roos TB, Porter DM, Sorenson GD. Cell-free DNA in human blood plasma: length measurements in patients with pancreatic cancer and healthy controls. Pancreas. 1998;17(1):89-97. https://doi.org/10.1097/00006676-199807000-00012

60. Lo YM, Chan KC, Sun H, Chen EZ, Jiang P, Lun FM, et al. Maternal plasma DNA sequencing reveals the genome-wide genetic and mutational profile of the fetus. Sci Transl Med. 2010;2(61):61ra91. https://doi.org/10.1126/scitranslmed.3001720

61. Mouliere F, Chandrananda D, Piskorz AM, Moore EK, Morris J, Ahlborn LB, et al. Enhanced detection of circulating tumor DNA by fragment size analysis. Sci Transl Med. 2018;10(466). https://doi. org/10.1126/scitranslmed.aat4921

62. Arnold A, Cossman J, Bakhshi A, Jaffe ES, Waldmann TA, Korsmeyer SJ. Immunoglobulingene rearrangements as unique clonal markers in human lymphoid neoplasms. N Engl J Med. 1983;309(26):1593-9. https://doi.org/10.1056/NEJM198312293092601

63. Camus V, Stamatoullas A, Mareschal S, Viailly PJ, Sarafan-Vasseur N, Bohers E, et al. Detection and prognostic value of recurrent exportin 1 mutations in tumor and cell-free circulating DNA of patients with classical Hodgkin lymphoma. Haematologica. 2016;101(9):1094-101. https://doi.org/10.3324/ haematol.2016.145102

64. Rossi D, Diop F, Spaccarotella E, Monti S, Zanni M, Rasi S, et al. Diffuse large B-cell lymphoma genotyping on the liquid biopsy. Blood. 2017;129(14):1947-57. https://doi.org/10.1182/ blood-2016-05-719641

65. Alcoceba M, García-Álvarez M, Chillón MC, Jiménez C, Medina A, Antón A, et al. Liquid biopsy: a non-invasive approach for Hodgkin lymphoma genotyping. Br J Haematol. 2021. https://doi. org/10.1111/bjh.17719

66. Swerdlow SH, Campo E, Pileri SA, Harris NL, Stein H, Siebert R, et al. The 2016 revision of the World Health Organization (WHO) classification of lymphoid neoplasms. Blood. 2016. https://doi. org/10.1182/blood-2016-01-643569

67. Carbone A, Gloghini A, Aldinucci D, Gattei V, Dalla-Favera R, Gaidano G. Expression pattern of MUMI/IRF4 in the spectrum of pathology of Hodgkin's disease. Br J Haematol. 2002;117(2):366-72. https://doi.org/10.1046/j.1365-2141.2002.03456.x

68. Reichel JB, McCormick J, Fromm JR, Elemento O, Cesarman E, Roshal M. Flow-sorting and Exome Sequencing of the Reed-Sternberg Cells of Classical Hodgkin Lymphoma. J Vis Exp. 2017(124). https://doi.org/10.3791/54399

69. Best MG, Sol N, Kooi I, Tannous J, Westerman BA, Rustenburg F, et al. RNA-Seq of Tumor-Educated Platelets Enables Blood-Based Pan-Cancer, Multiclass, and Molecular Pathway Cancer Diagnostics. Cancer Cell. 2015;28(5):666-76. https://doi.org/10.1016/j.ccell.2015.09.018

70. Best MG, Sol N, In 't Veld S, Vancura A, Muller M, Niemeijer AN, et al. Swarm IntelligenceEnhanced Detection of Non-Small-Cell Lung Cancer Using Tumor-Educated Platelets. Cancer Cell. 2017;32(2):238-52.e9. https://doi.org/10.1016/j.ccell.2017.07.004

71. Drees EEE, Roemer MGM, Groenewegen NJ, Perez-Boza J, van Eijndhoven MAJ, Prins LI, et al. Extracellular vesicle miRNA predict FDG-PET status in patients with classical Hodgkin Lymphoma. J Extracell Vesicles. 2021;10(9):e12121. https://doi.org/10.1002/jev2.12121

72. Jongen-Lavrencic M, Grob T, Hanekamp D, Kavelaars FG, Al Hinai A, Zeilemaker A, et al. Molecular Minimal Residual Disease in Acute Myeloid Leukemia. N Engl J Med. 2018;378(13):1189-99. https:// doi.org/10.1056/NEJMoal716863 
73. Dillon R, Potter N, Freeman S, Russell N. How we use molecular minimal residual disease (MRD) testing in acute myeloid leukaemia (AML). Br J Haematol. 2021;193(2):231-44. https://doi.org/10.1111/ bjh. 17185

74. Schaapveld M, Aleman BM, van Eggermond AM, Janus CP, Krol AD, van der Maazen RW, et al. Second Cancer Risk Up to 40 Years after Treatment for Hodgkin's Lymphoma. N Engl J Med. 2015;373(26):2499-511. https://doi.org/10.1056/NEJMoal505949

75. Armitage JO. Early-Stage Hodgkin's Lymphoma. New England Journal of Medicine. 2010;363(7):653-62. https://doi.org/10.1056/NEJMral003733

76. Ansell SM. Hodgkin lymphoma: 2018 update on diagnosis, risk-stratification, and management. Am J Hematol. 2018;93(5):704-15. https://doi.org/10.1002/ajh.25071

77. Filippi AR, Levis M, Parikh R, Hoppe B. Optimal Therapy for Early-Stage Hodgkin's Lymphoma: Risk Adapting, Response Adapting, and Role of Radiotherapy. Curr Oncol Rep. 2017;19(5):34. https://doi. org/10.1007/s11912-017-0592-7

78. Cerci JJ, Pracchia LF, Linardi CC, Pitella FA, Delbeke D, Izaki M, et al. 18F-FDG PET after 2 cycles of ABVD predicts event-free survival in early and advanced Hodgkin lymphoma. J Nucl Med. 2010;51(9):1337-43. https://doi.org/10.2967/jnumed.109.073197

79. Zaucha JM, Malkowski B, Chauvie S, Subocz E, Tajer J, Kulikowski W, et al. The predictive role of interim PET after the first chemotherapy cycle and sequential evaluation of response to ABVD in Hodgkin's lymphoma patients-the Polish Lymphoma Research Group (PLRG) Observational Study. Ann Oncol. 2017;28(12):3051-7. https://doi.org/10.1093/annonc/mdx524

80. Radford J, Illidge T, Counsell N, Hancock B, Pettengell R, Johnson P, et al. Results of a Trial of PET-Directed Therapy for Early-Stage Hodgkin's Lymphoma. New England Journal of Medicine. 2015;372(17):1598-607. https://doi.org/10.1056/NEJMoal408648

81. Andre M, Girinsky T, Federico M, Reman O, Fortpied V, Gotti M, et al. Early Positron Emission Tomography Response-Adapted Treatment in Stage I and II Hodgkin Lymphoma: Final Results of the Randomized EORTC/LYSA/FIL H10 Trial. Journal of Clinical Oncology. 2017;35(16):1786-94. https://doi.org/10.1200/JCO.2016.68.6394

82. Raemaekers JM, Andre MP, Federico M, Girinsky T, Oumedaly R, Brusamolino E, et al. Omitting radiotherapy in early positron emission tomography-negative stage I/II Hodgkin lymphoma is associated with an increased risk of early relapse: Clinical results of the preplanned interim analysis of the randomized EORTC/LYSA/FIL H10 trial. J Clin Oncol. 2014;32(12):1188-94. https://doi. org/10.1200/JCO.2013.51.9298

83. LaCasce A, Dockter T, Ruppert A, Kostakoglu L, Schöder H, Hsi E, et al. CALGB 50801 (Alliance): PET adapted therapy in bulky stage I/II classic Hodgkin lymphoma (cHL). Journal of Clinical Oncology. 2021;39(15_suppl):7507-. https://doi.org/10.1200/JCO.2021.39.15_suppl.7507

84. Kumar A, Casulo C, Advani RH, Budde E, Barr PM, Batlevi CL, et al. Brentuximab Vedotin Combined With Chemotherapy in Patients With Newly Diagnosed Early-Stage, Unfavorable-Risk Hodgkin Lymphoma. J Clin Oncol. 2021;39(20):2257-65. https://doi.org/10.1200/JCO.21.00108

85. Borchmann P, Plütschow A, Kobe C, Greil R, Meissner J, Topp MS, et al. PET-guided omission of radiotherapy in early-stage unfavourable Hodgkin lymphoma (GHSG HD17): a multicentre, openlabel, randomised, phase 3 trial. Lancet Oncol. 2021;22(2):223-34. https://doi.org/10.1016/ S1470-2045(20)30601-X

86. Bröckelmann PJ, Goergen H, Keller U, Meissner J, Ordemann R, Halbsguth TV, et al. Efficacy of Nivolumab and AVD in Early-Stage Unfavorable Classic Hodgkin Lymphoma: The Randomized Phase 2 German Hodgkin Study Group NIVAHL Trial. JAMA Oncol. 2020;6(6):872-80. https://doi. org/10.1001/jamaoncol.2020.0750

87. Skoetz N, Trelle S, Rancea M, Haverkamp H, Diehl V, Engert A, et al. Effect of initial treatment strategy on survival of patients with advanced-stage Hodgkin's lymphoma: a systematic review and network meta-analysis. Lancet Oncol. 2013;12(13):70341-3. https://doi.org/10.1016/ S1470-2045(13)70341-3

88. André MPE, Carde P, Viviani S, Bellei M, Fortpied C, Hutchings M, et al. Long-term overall survival and toxicities of ABVD vs BEACOPP in advanced Hodgkin lymphoma: A pooled analysis of four randomized trials. Cancer Med. 2020;9(18):6565-75. https://doi.org/10.1002/cam4.3298 
89. Zaucha JM, Chauvie S, Zaucha R, Biggii A, Gallamini A. The role of PET/CT in the modern treatment of Hodgkin lymphoma. Cancer Treat Rev. 2019;77:44-56. https://doi.org/10.1016/j.ctrv.2019.06.002

90. Gallamini A, Tarella C, Viviani S, Rossi A, Patti C, Mule A, et al. Early Chemotherapy Intensification With Escalated BEACOPP in Patients With Advanced-Stage Hodgkin Lymphoma With a Positive Interim Positron Emission Tomography/Computed Tomography Scan After Two ABVD Cycles: Long-Term Results of the GITIL/FIL HD 0607 Trial. J Clin Oncol. 2018;36(5):454-62. https://doi. org/10.1200/JCO.2017.75.2543

91. Trotman J, Fosså A, Federico M, Stevens L, Kirkwood A, Clifton-Hadley L, et al. RESPONSEADJUSTED THERAPY FOR ADVANCED HODGKIN LYMPHOMA (RATHL) TRIAL: LONGER FOLLOW UP CONFIRMS EFFICACY OF DE-ESCALATION AFTER a NEGATIVE INTERIM PET SCAN (CRUK/07/033). Hematological Oncology. 2017;35:65-7. https://doi.org/10.1002/ hon.2437_53

92. Eichenauer DA, Aleman BMP, Andre M, Federico M, Hutchings M, Illidge T, et al. Hodgkin lymphoma: ESMO Clinical Practice Guidelines for diagnosis, treatment and follow-up. Ann Oncol. 2018;29(Suppl 4):iv19-iv29. https://doi.org/10.1093/annonc/mdy080

93. Straus DJ, Długosz-Danecka M, Connors JM, Alekseev S, Illés Á, Picardi M, et al. Brentuximab vedotin with chemotherapy for stage III or IV classical Hodgkin lymphoma (ECHELON-1): 5-year update of an international, open-label, randomised, phase 3 trial. Lancet Haematol. 2021;8(6):e410-e21. https://doi.org/10.1016/S2352-3026(21)00102-2

94. Ramchandren R, Domingo-Domènech E, Rueda A, Trněný M, Feldman TA, Lee HJ, et al. Nivolumab for Newly Diagnosed Advanced-Stage Classic Hodgkin Lymphoma: Safety and Efficacy in the Phase II CheckMate 205 Study. J Clin Oncol. 2019;37(23):1997-2007. https://doi.org/10.1200/JCO.19.00315

95. Allen PB, Savas H, Evens AM, Advani RH, Palmer B, Pro B, et al. Pembrolizumab followed by AVD in untreated early unfavorable and advanced-stage classical Hodgkin lymphoma. Blood. 2021;137(10):1318-26. https://doi.org/10.1182/blood.2020007400

96. Rossi C, Gilhodes J, Maerevoet M, Herbaux C, Morschhauser F, Brice P, et al. Efficacy of chemotherapy or chemo-anti-PD-1 combination after failed anti-PD-1 therapy for relapsed and refractory Hodgkin lymphoma: A series from Lysa centers. Am J Hematol. 2018. https://doi.org/10.1002/ajh.25154

97. Carreau NA, Pail O, Armand P, Merryman R, Advani RH, Spinner MA, et al. Checkpoint Blockade Treatment May Sensitize Hodgkin Lymphoma to Subsequent Therapy. Oncologist. 2020;25(10):878-85. https://doi.org/10.1634/theoncologist.2020-0167

98. Linch DC, Winfield D, Goldstone AH, Moir D, Hancock B, McMillan A, et al. Dose intensification with autologous bone-marrow transplantation in relapsed and resistant Hodgkin's disease: results of a BNLI randomised trial. Lancet. 1993;341(8852):1051-4. https://doi.org/10.1016/0140-6736(93)92411-L

99. Schmitz N, Pfistner B, Sextro M, Sieber M, Carella AM, Haenel M, et al. Aggressive conventional chemotherapy compared with high-dose chemotherapy with autologous haemopoietic stemcell transplantation for relapsed chemosensitive Hodgkin's disease: a randomised trial. Lancet. 2002;359(9323):2065-71. https://doi.org/10.1016/S0140-6736(02)08938-9

100. Moskowitz CH, Matasar MJ, Zelenetz AD, Nimer SD, Gerecitano J, Hamlin P, et al. Normalization of pre-ASCT, FDG-PET imaging with second-line, non-cross-resistant, chemotherapy programs improves event-free survival in patients with Hodgkin lymphoma. Blood. 2012;119(7):1665-70. https://doi.org/10.1182/blood-2011-10-388058

101. Moskowitz CH, Nademanee A, Masszi T, Agura E, Holowiecki J, Abidi MH, et al. Brentuximab vedotin as consolidation therapy after autologous stem-cell transplantation in patients with Hodgkin's lymphoma at risk of relapse or progression (AETHERA): a randomised, double-blind, placebo-controlled, phase 3 trial. The Lancet. 2015;385(9980):1853-62. https://doi.org/10.1016/ S0140-6736(15)60165-9

102. LaCasce AS, Bociek RG, Sawas A, Caimi P, Agura E, Matous J, et al. Brentuximab vedotin plus bendamustine: a highly active first salvage regimen for relapsed or refractory Hodgkin lymphoma. Blood. 2018;132(1):40-8. https://doi.org/10.1182/blood-2017-11-815183

103. Garcia-Sanz R, Sureda A, de la Cruz F, Canales M, Gonzalez AP, Pinana JL, et al. Brentuximab vedotin and ESHAP is highly effective as second-line therapy for Hodgkin lymphoma patients (long-term results of a trial by the Spanish GELTAMO Group). Ann Oncol. 2019;30(4):612-20. https://doi. org/10.1093/annonc/mdz009 
104. Kersten MJ, Driessen J, Zijlstra JM, Plattel WJ, Morschhauser F, Lugtenburg PJ, et al. Combining brentuximab vedotin with dexamethasone, high-dose cytarabine and cisplatin as salvage treatment in relapsed or refractory Hodgkin lymphoma: the phase II HOVON/LLPC Transplant BRaVE study. Haematologica. 2021;106(4):1129-37. https://doi.org/10.3324/haematol.2019.243238

105. Advani R, Moskowitz AJ, Bartlett NL, Vose J, Ramchandren R, Feldman T, et al. Brentuximab vedotin in combination with nivolumab in relapsed or refractory Hodgkin lymphoma: 3-year study results. Blood. 2021. https://doi.org/10.1182/blood.2020009178

106. Brockelmann PJ, Muller H, Casasnovas O, Hutchings M, von Tresckow B, Jurgens M, et al. Risk factors and a prognostic score for survival after autologous stem-cell transplantation for relapsed or refractory Hodgkin lymphoma. Ann Oncol. 2017;28(6):1352-8. https://doi.org/10.1093/annonc/mdx072

107. Moskowitz CH, Walewski J, Nademanee A, Masszi T, Agura E, Holowiecki J, et al. Five-year PFS from the AETHERA trial of brentuximab vedotin for Hodgkin lymphoma at high risk of progression or relapse. Blood. 2018;132(25):2639-42. https://doi.org/10.1182/blood-2018-07-861641

108. Armand P, Chen YB, Redd RA, Joyce RM, Bsat J, Jeter E, et al. PD-1 blockade with pembrolizumab for classical Hodgkin lymphoma after autologous stem cell transplantation. Blood. 2019;134(1):22-9. https://doi.org/10.1182/blood.2019000215

109. Herrera AF, Chen L, Nieto Y, Holmberg L, Johnston PB, Mei M, et al. Consolidation with Nivolumab and Brentuximab Vedotin after Autologous Hematopoietic Cell Transplantation in Patients with High-Risk Hodgkin Lymphoma. Blood. 2020;136(Supplement 1):19-20. https://doi.org/10.1182/ blood-2020-136384

110. Sibon D, Morschhauser F, Resche-Rigon M, Ghez D, Dupuis J, Marçais A, et al. Single or tandem autologous stem-cell transplantation for first-relapsed or refractory Hodgkin lymphoma: 10-year follow-up of the prospective H96 trial by the LYSA/SFGM-TC study group. Haematologica. 2016;101(4):474-81. https://doi.org/10.3324/haematol.2015.136408

111. Bazarbachi A, Boumendil A, Sr., Finel H, Sr., Khvedelidze I, Romejko-Jarosinska J, Tanase AD, et al. Evolution of Outcome over Time for Relapsed Hodgkin Lymphoma after Autologous Stem Cell Transplant: Improved Survival for Early Relapse in Recent Years. Blood. 2020;136(Supplement 1):9-10. https://doi.org/10.1182/blood-2020-138546

112. Younes A, Gopal AK, Smith SE, Ansell SM, Rosenblatt JD, Savage KJ, et al. Results of a Pivotal Phase II Study of Brentuximab Vedotin for Patients With Relapsed or Refractory Hodgkin's Lymphoma. Journal of Clinical Oncology. 2012;30(18):2183-9. https://doi.org/10.1200/JCO.2011.38.0410

113. Chen R, Gopal AK, Smith SE, Ansell SM, Rosenblatt JD, Savage KJ, et al. Five-year survival and durability results of brentuximab vedotin in patients with relapsed or refractory Hodgkin lymphoma. Blood. 2016;128(12):1562-6. https://doi.org/10.1182/blood-2016-02-699850

114. Kuruvilla J, Ramchandren R, Santoro A, Paszkiewicz-Kozik E, Gasiorowski R, Johnson NA, et al. Pembrolizumab versus brentuximab vedotin in relapsed or refractory classical Hodgkin lymphoma (KEYNOTE-204): an interim analysis of a multicentre, randomised, open-label, phase 3 study. Lancet Oncol. 2021;22(4):512-24. https://doi.org/10.1016/S1470-2045(21)00005-X

115. Roemer MGM, Advani RH, Ligon AH, Natkunam Y, Redd RA, Homer H, et al. PD-L1 and PD-L2 Genetic Alterations Define Classical Hodgkin Lymphoma and Predict Outcome. Journal of Clinical Oncology. 2016. https://doi.org/10.1200/JCO.2016.66.4482

116. Chen R, Zinzani PL, Fanale MA, Armand P, Johnson NA, Brice P, et al. Phase II Study of the Efficacy and Safety of Pembrolizumab for Relapsed/Refractory Classic Hodgkin Lymphoma. J Clin Oncol. 2017;35(19):2125-32. https://doi.org/10.1200/JCO.2016.72.1316

117. Sureda A, Genadieva Stavrik S, Boumendil A, Finel H, Khvedelidze I, Dietricht S, et al. Changes in patients population and characteristics of hematopoietic stem cell transplantation for relapsed/refractory Hodgkin lymphoma: an analysis of the Lymphoma Working Party of the EBMT. Bone Marrow Transplant. 2020;55(11):2170-9. https://doi.org/10.1038/s41409-020-0929-y

118. Martínez C, Gayoso J, Canals C, Finel H, Peggs K, Dominietto A, et al. Post-Transplantation Cyclophosphamide-Based Haploidentical Transplantation as Alternative to Matched Sibling or Unrelated Donor Transplantation for Hodgkin Lymphoma: A Registry Study of the Lymphoma Working Party of the European Society for Blood and Marrow Transplantation. J Clin Oncol. 2017;35(30):3425-32. https://doi.org/10.1200/JCO.2017.72.6869 
119. Bartlett NL, Herrera AF, Domingo-Domenech E, Mehta A, Forero-Torres A, Garcia-Sanz R, et al. A phase 1 b study of AFM13 in combination with pembrolizumab in patients with relapsed or refractory Hodgkin lymphoma. Blood. 2020;136(21):2401-9. https://doi.org/10.1182/blood.2019004701

120. Hamadani M, Collins GP, Caimi PF, Samaniego F, Spira A, Davies A, et al. Camidanlumab tesirine in patients with relapsed or refractory lymphoma: a phase 1 , open-label, multicentre, doseescalation, dose-expansion study. Lancet Haematol. 2021;8(6):e433-e45. https://doi.org/10.1016/ S2352-3026(21)00103-4

121. Ramos CA, Grover NS, Beaven AW, Lulla PD, Wu MF, Ivanova A, et al. Anti-CD30 CAR-T Cell Therapy in Relapsed and Refractory Hodgkin Lymphoma. J Clin Oncol. 2020:Jco2001342. https:// doi.org/10.1200/JCO.20.01342

122. Csizmar CM, Ansell SM. Engaging the Innate and Adaptive Antitumor Immune Response in Lymphoma. Int J Mol Sci. 2021;22(7). https://doi.org/10.3390/ijms22073302

123. Green MR, Monti S, Rodig SJ, Juszczynski P, Currie T, O’Donnell E, et al. Integrative analysis reveals selective 9p24.1 amplification, increased PD-1 ligand expression, and further induction via JAK2 in nodular sclerosing Hodgkin lymphoma and primary mediastinal large B-cell lymphoma. Blood. 2010;116(17):3268-77. https://doi.org/10.1182/blood-2010-05-282780

124. Cader FZ, Schackmann RCJ, Hu X, Wienand K, Redd R, Chapuy B, et al. Mass cytometry of Hodgkin lymphoma reveals a CD4(+) regulatory T-cell-rich and exhausted T-effector microenvironment. Blood. 2018;132(8):825-36. https://doi.org/10.1182/blood-2018-04-843714

125. Ansell SM, Lesokhin AM, Borrello I, Halwani A, Scott EC, Gutierrez M, et al. PD-1 blockade with nivolumab in relapsed or refractory Hodgkin's lymphoma. N Engl J Med. 2015;372(4):311-9. https:// doi.org/10.1056/NEJMoal411087

126. Armand P, Engert A, Younes A, Fanale M, Santoro A, Zinzani PL, et al. Nivolumab for Relapsed/ Refractory Classic Hodgkin Lymphoma After Failure of Autologous Hematopoietic Cell Transplantation: Extended Follow-Up of the Multicohort Single-Arm Phase II CheckMate 205 Trial. J Clin Oncol. 2018;36(14):1428-39. https://doi.org/10.1200/JCO.2017.76.0793

127. Ansell SM, Brockelmann PJ, von Keudell G, Lee HJ, Santoro A, Zinzani PL, et al. Nivolumab for relapsed or refractory classical Hodgkin lymphoma after autologous transplantation: 5-year overall survival from the phase 2 CheckMate 205 study. Proc ICML. 2021. https://doi.org/10.1002/ hon.74_2879

128. Chen R, Zinzani PL, Lee HJ, Armand P, Johnson NA, Brice P, et al. Pembrolizumab in relapsed or refractory Hodgkin lymphoma: 2-year follow-up of KEYNOTE-087. Blood. 2019;134(14):1144-53. https://doi.org/10.1182/blood.2019000324

129. Herrera AF, Moskowitz AJ, Bartlett NL, Vose JM, Ramchandren R, Feldman TA, et al. Interim results of brentuximab vedotin in combination with nivolumab in patients with relapsed or refractory Hodgkin lymphoma. Blood. 2018;131(11):1183-94. https://doi.org/10.1182/blood-2017-10-811224

130. Diefenbach CS, Hong F, Ambinder RF, Cohen JB, Robertson MJ, David KA, et al. Ipilimumab, nivolumab, and brentuximab vedotin combination therapies in patients with relapsed or refractory Hodgkin lymphoma: phase 1 results of an open-label, multicentre, phase 1/2 trial. Lancet Haematol. 2020;7(9):e660-e70. https://doi.org/10.1016/\$2352-3026(20)30221-0

131. Cheson BD, Bartlett NL, LaPlant B, Lee HJ, Advani RJ, Christian B, et al. Brentuximab vedotin plus nivolumab as first-line therapy in older or chemotherapy-ineligible patients with Hodgkin lymphoma (ACCRU): a multicentre, single-arm, phase 2 trial. Lancet Haematol. 2020;7(11):e808-el5. https:// doi.org/10.1016/S2352-3026(20)30275-1

132. Ramchandren R, Domingo-Domenech E, Rueda A, Trneny M, Feldman TA, Lee HJ, et al. Nivolumab for Newly Diagnosed Advanced-Stage Classic Hodgkin Lymphoma: Safety and Efficacy in the Phase II CheckMate 205 Study. J Clin Oncol. 2019;37(23):1997-2007. https://doi.org/10.1200/JCO.19.00315

133. Advani R, Flinn I, Popplewell L, Forero A, Bartlett NL, Ghosh N, et al. CD47 Blockade by Hu5F9-G4 and Rituximab in Non-Hodgkin's Lymphoma. The New England journal of medicine. 2018;379(18):1711-21. https://doi.org/10.1056/NEJMoal807315

134. Ansell SM, Minnema MC, Johnson P, Timmerman JM, Armand P, Shipp MA, et al. Nivolumab for Relapsed/Refractory Diffuse Large B-Cell Lymphoma in Patients Ineligible for or Having Failed Autologous Transplantation: A Single-Arm, Phase II Study. J Clin Oncol. 2019;37(6):481-9. https:// doi.org/10.1200/JCO.18.00766 
135. Patel K, Ramchandren R, Maris M, Lesokhin AM, von Keudell GT, Cheson BD, et al. Investigational CD47-blocker TTI-662 shows single-agent activity in patients with advanced relapsed or refractory lymphoma: update from the onoing first-in-human dose escalation study. Blood. 2020;136:abstract \#1191. https://doi.org/10.1182/blood-2020-136607 